\title{
Effective Use of Magnetometer Feedback for Smart Projectile Applications
}

\author{
JONATHAN ROGERS and MARK COSTELLO \\ Georgia Institute of Technology, Atlanta, GA, 30332 \\ THOMAS HARKINS \\ US Army Research Laboratory, Aberdeen, MD, 21001 \\ MOSHE HAMAOUI \\ Data Matrix Solutions, Inc., Aberdeen, MD, 21001 \\ Received September 2010; Revised March 2011
}

\begin{abstract}
The use of magnetometers for orientation estimation on rapidly-spinning flight bodies is analyzed. Specifically, the effect of spin-induced magnetic field distortion is discussed, with particular attention to its implications for magnetometer-based orientation estimation. First, the nature of spin-induced field distortion is described and it is shown that, if not properly accounted for, distortion can lead to significant estimation errors in artillery projectiles. Then, an orientation estimator is constructed driven by magnetometer, gyroscope, and GPS feedback. A novel feature of this algorithm is its compensation for spin-induced distortion of the Earth's field. The algorithm also incorporates in-flight magnetometer calibration performed simultaneously with projectile orientation estimation. The comprehensive algorithm is built as a coupled set of Extended Kalman filters. Observability of the estimation problem is discussed and unobservable modes are identified. Finally, example results and Monte Carlo simulations compare estimation performance to algorithms which neglect spin-induced distortion effects or do not perform in-flight calibration. These results demonstrate that magnetometer-based systems on-board spinning projectiles should incorporate corrections for field distortion, and that overall accuracy is greatly enhanced by performing in-flight calibration.
\end{abstract}

\section{INTRODUCTION}

The availability of low-cost, lightweight sensors and digital microprocessors has enabled weapons designers to equip artillery projectiles with full guidance and control capability. However, in comparison to electronics packages on-board missiles, projectile sensor suites and guidance units must be able to withstand large acceleration loads at launch and high spin rates while maintaining low cost. One challenge routinely faced by designers has been the development of attitude estimators for these vehicles. While accurate attitude information is critical to control system performance, it is often impossible to obtain by integrating outputs from low-cost rate sensors. One common solution is to use magnetometer measurements to provide periodic updates to integrated rate sensor data. Magnetometers are attractive due to their low power requirements, rugged construction, and low cost.

Navigation: Journal of The Institute of Navigation

Vol. 58, No. 3, Fall 2011

Printed in the U.S.A.
The use of magnetometers to obtain orientation information is not a new idea and has been used for many types of flight vehicles, from satellites to UAVs. Typically, an algorithm is employed to determine a solution for the direction cosines matrix (DCM), Euler angles, or quaternions of the vehicle based on a set of magnetometer measurements. Wahba [1, 2] initially proposed a batch least squares technique for determining the DCM of a satellite based on vector measurements. Other researchers proposed purely deterministic methods [3] and recursive algorithms [4] for computing the DCM. BarItzhak and Oshman [5] extended this work by developing a method for recursively estimating quaternions from a set of vector measurements. Similar algorithms [6] were developed for Euler angle determination. Most recently, Mortari [7] has shown that when using more than two vector measurements, the optimal Euler axis and the principle Euler angle can be obtained separately without iterative procedures. Psiaki [8] developed a magnetometer-only attitude and angular rate estimator for low-cost spacecraft and showed reasonable performance using flight data. 
While much of the above research has been directed toward spacecraft applications, several investigators in the projectile community have recently developed algorithms specifically tailored to smart projectiles. Ohlmeyer, Fraysse, and Pepitone [9] incorporated magnetometers in a low-cost INS along with accelerometers and GPS. Magnetometer bias was estimated in-flight to improve performance. Wilson [10] also proposed the use of magnetometers as the primary attitude sensor on-board smart projectiles. He showed that complete attitude solutions could be obtained only by combining magnetometers with additional sensors such as accelerometers or solar sensors. Most recently, Lee et al. [11] developed a roll attitude estimator for smart munitions using magnetometers based on an unscented Kalman filter.

Two recurring problems have consistently hindered efforts to implement magnetometer-based estimators on-board guided artillery shells. The first is that distortion and attenuation of the Earth's magnetic field can be significant inside the body of a spinning projectile due to the formation of eddy currents within the conducting metallic body. Harkins [12] has explored this phenomenon experimentally, concluding that these effects can lead to significant error in magnetometer-based estimators if proper compensation is not used. The second, and perhaps less application-specific, is that bias, scale factor, and misalignment errors can often have significant detrimental impact on overall attitude estimation performance. Calibration values, especially bias, can change during launch or in flight. Several techniques have previously been developed to mitigate this problem by performing on-line estimation of these nuisance parameters (autocalibration). Lerner and Shuster [13] first developed a method to estimate magnetometer nuisance parameters on-board spacecraft given a priori knowledge of attitude. Alonso and Shuster [14-16] have proposed so-called "attitude independent" autocalibration methods for spacecraft that rely on changes in the Earth's magnetic field magnitude over one orbit cycle. Crassidis et al. [17] expanded on this work, developing three algorithms to perform real-time magnetometer calibration based on observed differences in field magnitude. Most recently, Gebre-Egziabher [18] developed an autocalibration algorithm for UAVs by fitting an ellipsoid to measured magnetic field data. Magnetometer data used to define this ellipsoid is generated by rotating the vehicle through prescribed turns during the calibration process.

Previously-developed autocalibration algorithms are for the most part unsuitable for projectile applications for several reasons. First, the Earth's magnetic field does not change enough throughout flight to employ attitude-independent solutions. Second, prescribed calibration maneuvers such as those outlined in [18] are typically not an option during projectile flight. Standard pre-flight sensor calibration procedures using reference sensors are too expensive given the low-cost nature of gunlaunched munitions, and cannot account for calibration changes after launch due to large shocks. Although it may be possible to develop sensors that maintain calibration through launch and do not require extensive calibration, cost considerations for projectile applications make this solution less attractive. Thus, new techniques are required to perform in-flight estimation of as many nuisance parameters as possible.

The contributions of this article are twofold. First, an in-depth analysis of the problem of magnetic field distortion inside projectile bodies is presented. An analytical model for this field distortion is built and compared to experimental results. A new magnetometer model is built which incorporates this distortion, and is used to generate an Extended Kalman filter that estimates field distortion parameters. The second main contribution is the development of a coupled set of Extended Kalman filters that estimate projectile orientation, nuisance parameters, and distortion effects simultaneously. Using this filter, a direct analysis of the benefits of in-flight nuisance parameter and field distortion estimation is performed. This is accomplished through example and Monte Carlo simulations in which performance of estimators with and without the capability to perform in-flight nuisance and distortion parameter identification is directly compared. Results show a significant benefit to incorporating distortion effects and performing nuisance parameter estimation in flight. The article begins by describing magnetic field distortion within rapidly-spinning projectile bodies. Then, algorithms are developed to estimate distortion effects, magnetometer nuisance parameters, and projectile orientation simultaneously. An observability analysis of the entire estimation problem is then performed and unobservable parameters are identified. Finally, simulation results show that attitude estimation performance is almost always improved when distortion effects are incorporated and nuisance parameter estimation is performed.

\section{SPIN-INDUCED MAGNETIC FIELD DISTORTION}

When a conductive body is subjected to a changing magnetic field, eddy current effects occur inside the body. Equivalently, a spinning cylinder im7mersed in a transverse uniform magnetic field is essentially subject to two radial oscillating fields which are 90 degrees apart in space and time. By Faraday's law, these oscillating magnetic fields will induce electric fields according to 


$$
\nabla \times \vec{E}=-\frac{\partial \vec{B}}{\partial t}
$$

If the cylinder is conductive, eddy currents will be induced to flow along the length of the cylinder. These currents in turn give rise to a secondary magnetic field which, in superposition with the external field, creates a distorted magnetic field in and around the cylinder [19]. Because on-board electronics packages used for smart weapon guidance and control are located within a rapidly spinning projectile body, magnetometer-based orientation estimators must compensate for this field distortion to avoid significant error.

\section{Modeling Spin-Induced Distortion: Infinite Cylinder}

Maxwell's equations lead to a diffusion equation for the magnetic vector potential describing the time evolution of the magnetic field, given by

$$
\nabla^{2} \vec{A}=\mu \sigma \frac{\partial \vec{A}}{\partial t}
$$

where $\vec{A}$ is the magnetic vector potential, $\mu$ is the magnetic permeability, and $\sigma$ is the electrical conductivity. Note that in the above equation the simplifying assumption that the displacement current in Maxwell's equations is negligible is made, since projectile spin rates correspond to wavelengths that are orders of magnitude larger than typical projectile body dimensions. Recently, Ziolkowski and Grabowski [20] imposed appropriate boundary conditions on Equation (2) to obtain an analytic solution for the case of an infinite, hollow cylinder spinning in a uniform, transverse magnetic field. Figure 1 shows magnetic field lines for an infinite, nonmagnetic, conducting cylinder of inner radius 50 $\mathrm{mm}$ and outer radius $75 \mathrm{~mm}$ rotating counterclockwise at a rate of $20 \mathrm{rad} / \mathrm{s}$ placed in a constant transverse external field (computed using the expressions from reference [20]). Note that, for an infinitely-long conducting cylinder, the field inside the body is uniform (i.e., constant distortion angle throughout) and attenuated with respect to the external transverse field. Any axial component of the external field is unaffected for cylinders of infinite length.

\section{Modeling Spin-Induced Distortion: Finite Cylinder}

For cylinders of finite length, no closed-form solution for the magnetic field is possible. Thus, finite element methods must be employed to model magnetic field distortion inside rotating bodies of finite length. To analyze distortion effects for finite bodies, a finite element (FE) model was constructed using ANSYS software. First, for validation purposes, the FE model was used to generate the spin-

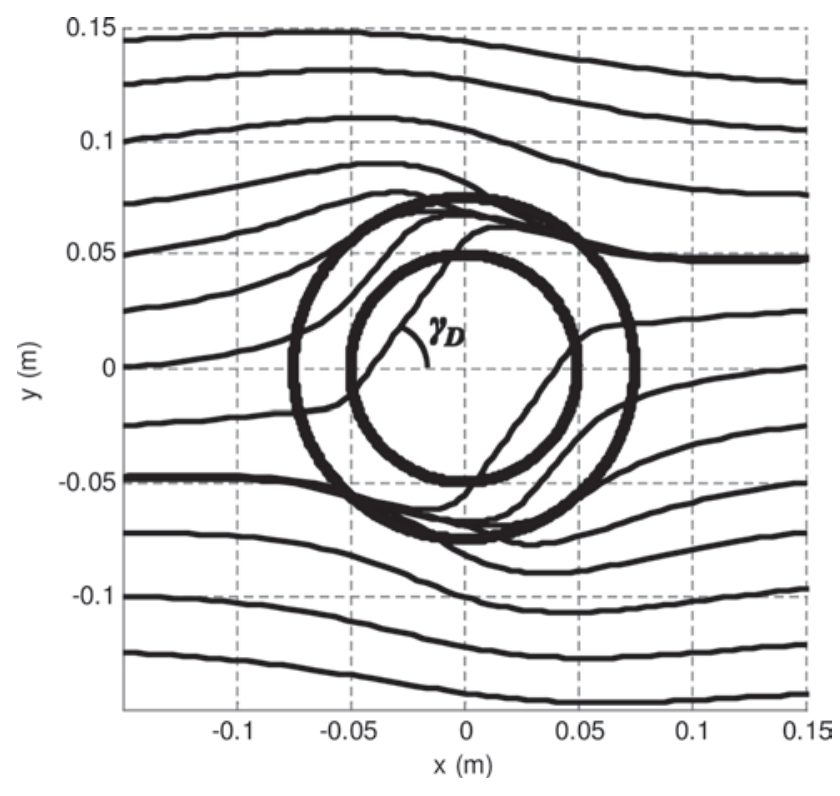

Fig. 1-Magnetic field lines for infinite cylinder rotating counterclockwise in an external magnetic field

distorted field for an infinite cylinder, and results were compared to those generated with the closedform solution. The magnetic fields generated by these two models matched to within $1 \%$. Then, the FE model considered a nonmagnetic hollow cylinder composed of $6061 \mathrm{Al}$, this time with inner and outer radii of approximately $50 \mathrm{~mm}$ and $57 \mathrm{~mm}$ respectively and length of $175 \mathrm{~mm}$. This finite-length cylinder was immersed in a uniform transverse magnetic field of $1 \mathrm{Gs}$ and spun at selected frequencies. Figure 2 shows example magnetic field results generated with this $\mathrm{FE}$ model for this finite cylinder spinning at a rate of $80 \mathrm{~Hz}$.

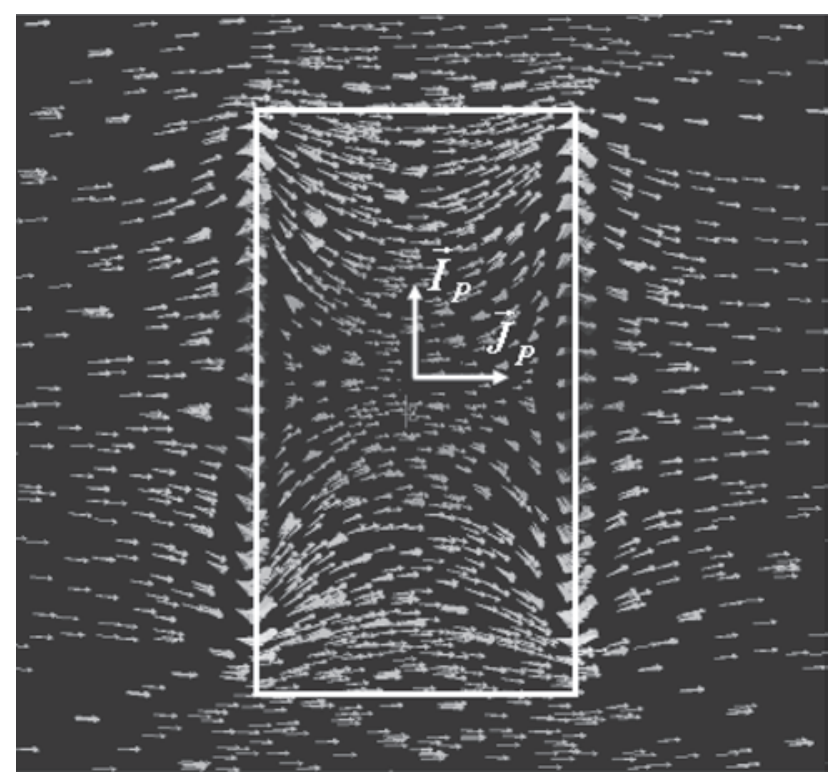

Fig. 2-Magnetic field vectors near a cylinder spinning at $80 \mathrm{~Hz}$, generated by FEA. The cylinder spins about $\vec{I}_{P}$ 
Figure 2 highlights some key differences between distortion effects from infinite- and finite-length cylinders. First, in contrast to the infinite-length case, the field inside finite-length cylinders is not uniform. Second, in the finite-length case, the distorted field inside the cylinder has a noticeable axial component. This axial component is zero along the spin axis and in the transverse plane of the centroid, but grows considerably near the edges of the cylinder.

Experiments were conducted to validate results from the FE model. A Helmholtz coil was used to generate a near-uniform rotating magnetic field near a cylinder composed of $6061 \mathrm{Al}$ with dimensions approximately equal to those described above. The experiment was performed at selected frequencies between 0 and $250 \mathrm{~Hz}$, and the magnetic field was measured approximately at the centroid of the cylinder.

In order to quantitatively compare results between the analytical model, FE model, and experiment, three variables are defined that describe spin-induced distortion: the attenuation factor $(A F)$, the transverse distortion angle, $\gamma_{D}$, and the induced axial component, $\zeta_{D}$. Attenuation factor $(A F)$ is a value representing the attenuation of the components of the Earth's field transverse to the projectile spin. Distortion angle $\left(\gamma_{D}\right)$ represents the change in direction of the transverse components of the field (as shown in Figure 1). The induced axial component $\left(\zeta_{D}\right)$ is a value between 0 and 1 that introduces distortion in the axial direction as a percentage of the overall transverse field strength. Let the components of the Earth's magnetic field vector inside the projectile body expressed in the body reference frame be given by $\tilde{\boldsymbol{m}}_{x \boldsymbol{D}}, \tilde{\boldsymbol{m}}_{\boldsymbol{y} \boldsymbol{D}}, \tilde{\boldsymbol{m}}_{z \boldsymbol{D}}$. Therefore,

$$
\begin{aligned}
\left\{\begin{array}{c}
\tilde{\boldsymbol{m}}_{x \boldsymbol{D}} \\
\tilde{\boldsymbol{m}}_{\boldsymbol{y} \boldsymbol{D}} \\
\tilde{\boldsymbol{m}}_{\boldsymbol{z} \boldsymbol{D}}
\end{array}\right\}= & {\left[\begin{array}{ccc}
1 & 0 & 0 \\
0 & c_{\gamma_{D}} & s_{\gamma_{D}} \\
0 & -s_{\gamma_{D}} & \boldsymbol{c}_{\gamma_{D}}
\end{array}\right]\left\{\begin{array}{c}
\tilde{\boldsymbol{m}}_{\boldsymbol{x}} \\
\tilde{\boldsymbol{m}}_{\boldsymbol{y}}(1-A F) \\
\tilde{\boldsymbol{m}}_{\boldsymbol{z}}(1-A F)
\end{array}\right\} } \\
& +\left\{\begin{array}{c}
\zeta_{D} \sqrt{\tilde{\boldsymbol{m}}_{\boldsymbol{y}}^{2}+\tilde{\boldsymbol{m}}_{\boldsymbol{z}}^{2}} \\
0 \\
0
\end{array}\right\}
\end{aligned}
$$

where $\tilde{\boldsymbol{m}}_{\boldsymbol{x}}, \tilde{\boldsymbol{m}}_{\boldsymbol{y}}, \tilde{\boldsymbol{m}}_{\boldsymbol{z}}$ are components of the Earth's magnetic field expressed in the body reference frame. Note that in Equation (3), and in the remainder of this article, $c_{\alpha}$ denotes $\cos (\alpha), s_{\alpha}$ denotes $\sin (\alpha)$, and $t_{\alpha}$ denotes $\tan (\alpha)$. Given both the nominal and distorted sets of magnetic field components, it is also possible to solve Equation (3) for $A F, \gamma_{D}$, and $\zeta_{D}$ such that

$$
A F=\frac{\sqrt{\tilde{\boldsymbol{m}}_{\boldsymbol{y}}^{2}+\tilde{\boldsymbol{m}}_{\boldsymbol{z}}^{2}}-\sqrt{\tilde{\boldsymbol{m}}_{\boldsymbol{y} \boldsymbol{D}}^{2}+\boldsymbol{m}_{z D}^{2}}}{\sqrt{\tilde{\boldsymbol{m}}_{\boldsymbol{y}}^{2}+\tilde{\boldsymbol{m}}_{\boldsymbol{z}}^{2}}}
$$

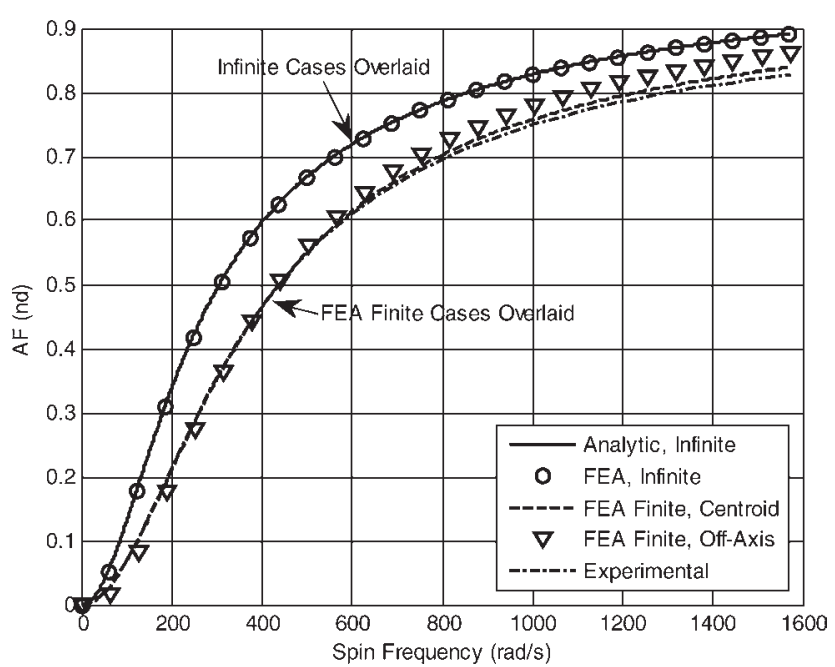

Fig. 3-Attenuation factor vs spin rate

$$
\begin{gathered}
\gamma_{D}=\tan ^{-1}\left(\frac{\tilde{\boldsymbol{m}}_{\boldsymbol{z} \boldsymbol{D}}}{\tilde{\boldsymbol{m}}_{\boldsymbol{y} \boldsymbol{D}}}\right)-\tan ^{-1}\left(\frac{\tilde{\boldsymbol{m}}_{\boldsymbol{z}}}{\tilde{\boldsymbol{m}}_{\boldsymbol{y}}}\right) \\
\zeta_{D}=\frac{\tilde{\boldsymbol{m}}_{\boldsymbol{x} \boldsymbol{D}}-\tilde{\boldsymbol{m}}_{\boldsymbol{x}}}{\sqrt{\tilde{\boldsymbol{m}}_{\boldsymbol{y}}^{2}+\tilde{\boldsymbol{m}}_{\boldsymbol{z}}^{2}}}
\end{gathered}
$$

Figures 3 and 4 show attenuation factor and distortion angle as a function of spin rate calculated by the analytic model and the FE model as well as values obtained from experiment. These figures show results for distortion parameters both at the centroid (both models and experiment) and at a selected example point away from the centroid (FE model only). The selected example point is located $34 \mathrm{~mm}$ in the radial direction and $25 \mathrm{~mm}$ in the longitudinal direction from the centroid of the cylinder. Furthermore, Figure 5 shows the induced axial component $\left(\zeta_{D}\right)$ as a function of spin rate at the selected example point generated by the FE model (experimental data at this example location is not currently available).

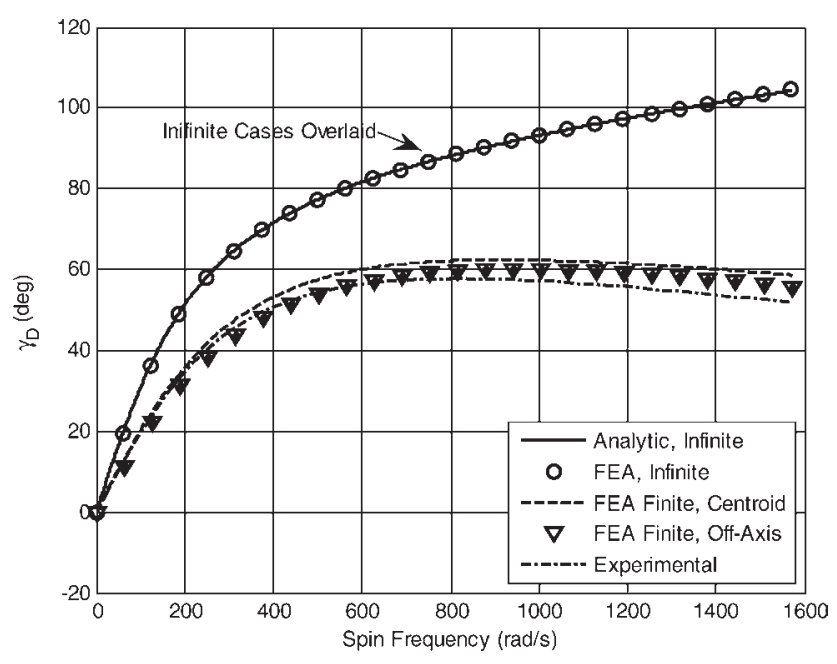

Fig. 4-Transverse distortion angle vs spin rate 


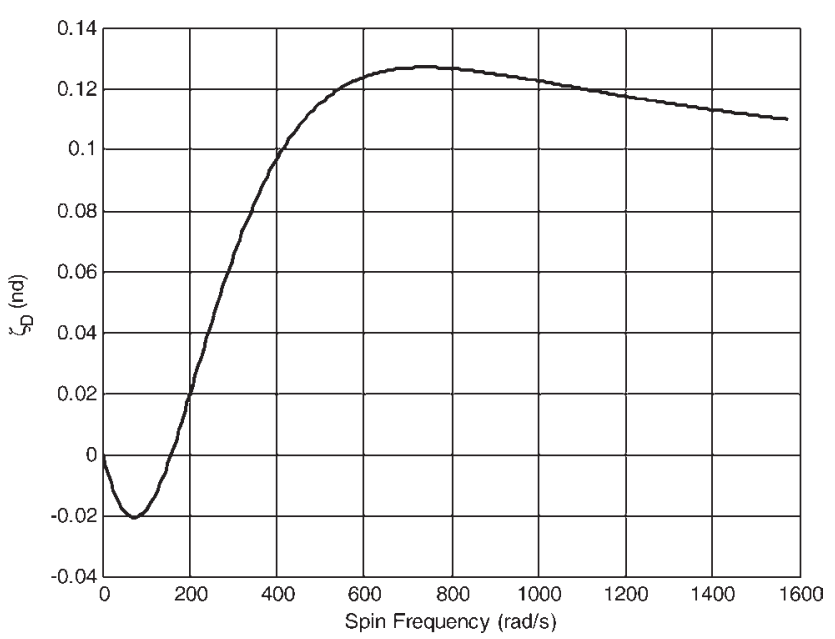

Fig. 5-Induced axial component vs spin rate at selected example point (FE model)

The results shown in Figures 3-5 demonstrate that all distortion parameters vary smoothly as a function of spin rate. Furthermore, even for projectiles exhibiting relatively low spin rates, these effects can be considerable. For spin rates between $40 \mathrm{rad} / \mathrm{s}$ and $120 \mathrm{rad} / \mathrm{s}$ (the range of roll rates for the example projectiles considered here), polynomial curve fits were performed to fit FEA data at the selected example point, and were found to produce reasonably accurate approximations. Note that a cubic fit proved adequate to describe $A F$ as a function of roll rate while a quadratic fit proved adequate to describe both $\gamma_{D}$ and $\zeta_{D}$ as a function of roll rate. Therefore,

$$
\begin{gathered}
A F \approx a_{3} p^{3}+a_{2} p^{2}+a_{1} p+a_{0} \\
\gamma_{D} \approx w_{2} p^{2}+w_{1} p+w_{0} \\
\zeta_{D}=z_{2} p^{2}+z_{1} p+z_{0}
\end{gathered}
$$

The coefficients in Equations (7)-(9) can be easily determined for a specific projectile configuration through a calibration process in which the projec- tile is rotated at various spin rates in the presence of a known external field, and the magnetic field within the projectile carefully measured at the magnetometer location.

With valid approximations for distortion parameters at the location of the magnetometer sensor, it is possible to compensate for and even estimate these distortion parameters in flight. The remainder of this article focuses on how magnetometers can be used effectively for real-time orientation estimation in spinning projectiles, and compares estimation performance to algorithms that neglect spin-induced distortion.

\section{ESTIMATOR DESIGN}

The projectile orientation estimator proposed here consists of three coupled Extended Kalman filters: one to estimate Euler angles; one to determine magnetometer scale factor, bias, and misalignment; and, one to determine magnetic field distortion parameters. These three filters are coupled as shown in Figure 6, creating a so-called "integrated filter" design. The three filters operate somewhat independently, with the nuisance parameter and distortion fit parameter estimation updated at specified intervals.

The choice to implement the three filters shown in Figure 6 as a coupled set of filters rather than as one large filter stems from several factors. First, note that because the derivatives of nuisance and distortion fit parameters are assumed to be zero, the integration portions of the filters naturally decouple. Second, the integrated filter provides a simple mechanism for turning off nuisance and distortion fit parameter estimation in poor estimation geometries, such as when flight occurs along the magnetic field vector. Third, the update rates required for Euler angle estimation are at least one order of magnitude higher than for nuisance and distortion fit parameter estimation, and thus signif-

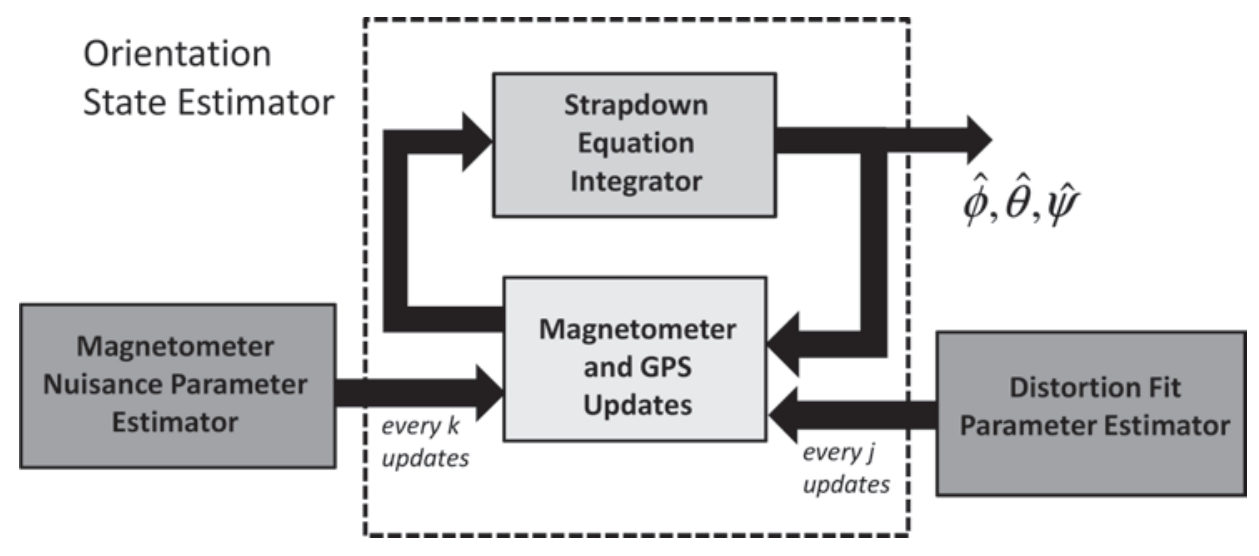

Fig. 6-Integrated filter schematic 
icant computation time can be saved by avoiding high-frequency updates to these parameters.

This section describes the design of each of the three coupled Extended Kalman filters (EKFs). Kalman filters generally use a process model to propagate state estimates and a measurement model to update state estimates whenever measurements are available. Note that the sensor suite considered here consists of a three-axis magnetometer, a threeaxis rate gyroscope, and GPS-derived inertial velocity feedback. Signals from the rate gyroscope are used directly to drive Euler angle dynamics, and thus scale factor errors, biases, and misalignments of the gyroscopes are not estimated.

The first section describes sensor and projectile geometry, while subsequent sections discuss the distortion fit, magnetometer nuisance parameter, and orientation state estimators, respectively. In the final section, an observability analysis of the entire problem is performed and unobservable states are removed.

\section{Sensor and Projectile Geometry}

Feedback from three sources are assumed to be available, namely a three-axis magnetometer (located near the projectile centerline), a three-axis rate gyro, and an inertial velocity estimator. In order to maintain as general a formulation as possible, the three-axis magnetometer is treated as three single-axis magnetometers. It can be shown that the number of misalignment angles to be estimated for three single-axis sensors is the same as for one three-axis sensor, and thus no estimation penalty is incurred by this treatment. The magnetometer's sensitive axis, denoted by $\vec{s}$, is described by two rotation angles and can be obtained by first rotating the $\vec{I}_{P}-\vec{J}_{P}$ plane by $\phi_{S}$ about $\vec{I}_{P}$, and then rotating $\vec{s}$ off the projectile centerline by $\psi_{S}$ as shown in Figure 7 . The output of a single axis magnetometer can be written as

$$
m^{*}=S\left(\tilde{\boldsymbol{m}}_{\boldsymbol{x}} c_{\psi_{S}}+\tilde{\boldsymbol{m}}_{\boldsymbol{y}} c_{\phi_{S}} \boldsymbol{s}_{\psi_{S}}+\tilde{\boldsymbol{m}}_{\boldsymbol{z}} s_{\phi_{S}} s_{\psi_{S}}+b+n\right)
$$

where $\tilde{\boldsymbol{m}}_{\boldsymbol{x}}, \tilde{\boldsymbol{m}}_{\boldsymbol{y}}, \tilde{\boldsymbol{m}}_{\boldsymbol{z}}$ are the components of the external magnetic field resolved into the body reference frame, $S$ is scale factor, $b$ represents bias, and $n$ represents zero-mean Gaussian white noise.

Angular rate feedback is provided by a three-axis rate gyroscope assumed to be aligned with the body axes. The output from the gyro is given by

$$
\begin{aligned}
\left\{\begin{array}{c}
\omega_{x}^{*} \\
\omega_{y}^{*} \\
\omega_{z}^{*}
\end{array}\right\}= & {\left[\begin{array}{ccc}
S_{G x} & c^{p q} & c^{p r} \\
c^{q p} & S_{G y} & c^{q r} \\
c^{r p} & c^{r q} & S_{G y}
\end{array}\right]\left\{\begin{array}{l}
p \\
q \\
r
\end{array}\right\}+\left\{\begin{array}{l}
b_{p} \\
b_{q} \\
b_{r}
\end{array}\right\} } \\
& +\left\{\begin{array}{l}
n_{p} \\
n_{q} \\
n_{r}
\end{array}\right\}
\end{aligned}
$$

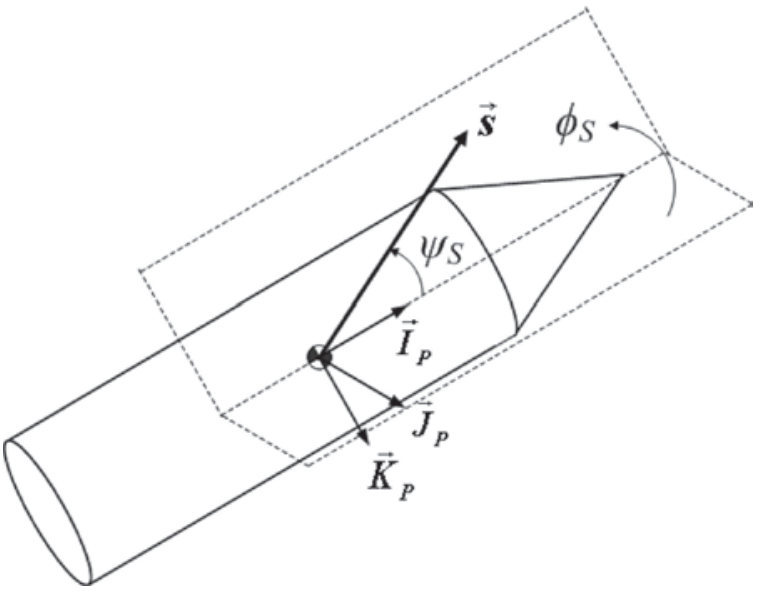

Fig. 7-Magnetometer sensitive axis definition

Note that for projectile applications, the requirement for low-cost sensors coupled with high spin rates prohibit use of pure angular rate integration for attitude estimation.

Estimation of all three Euler angles using only magnetometer and rate gyro feedback is not an observable problem if we are to avoid using angular rate integration to obtain one of the angles. Numerous techniques have been developed to complete the magnetometer-based orientation estimation problem, including use of derivatives of magnetometer signals, feedback from other less accurate roll sensors, or approximation of pitch and roll angle using inertial velocity feedback [10]. For projectile applications, the last technique proves to be an attractive option for two reasons. First, inertial velocity feedback is typically readily available anyway from GPS, an IMU, or a combination. Second, projectiles typically experience small angles of attack, even during maneuver, allowing pitch and roll to be determined from velocity with reasonable accuracy. Thus, given mass center velocity components in the inertial frame, $v_{x}, v_{y}, v_{z}$, and assuming zero angle of attack, direct measurements for pitch and yaw angle are possible, given by

$$
\theta_{m}=\sin ^{-1}\left(\frac{-v_{z}}{\sqrt{v_{x}^{2}+v_{y}^{2}+v_{z}^{2}}}\right) ; \psi_{m}=\tan ^{-1}\left(\frac{v_{y}}{v_{z}}\right)
$$

Note that the above expressions are only valid for small angles of attack. However, in the presence of angles of attack beyond a few degrees, $\theta_{m}$ and $\psi_{m}$ can be viewed as noisy measurements and subsequent error can be accounted for by proper tuning of filter gains.

\section{Distortion Fit Parameter Estimator}

With the knowledge that $A F$ is approximately a quadratic function of roll rate, and $\gamma_{D}$ and $\zeta_{D}$ are 
approximately cubic functions of roll rate, an Extended Kalman filter (EKF) is designed to estimate polynomial fit parameters in real time. First, note that the magnetometer equation now changes from the expression shown in Equation (10) to

$$
\begin{aligned}
m^{*}= & S\left(\tilde{\boldsymbol{m}}_{\boldsymbol{x}} c_{\psi_{S}}+\zeta_{D} c_{\psi_{S}} \sqrt{\tilde{\boldsymbol{m}}_{\boldsymbol{y}}^{2}+\tilde{\boldsymbol{m}}_{\boldsymbol{z}}^{2}}+b+n\right) \\
& +S(1-A F) c_{\gamma_{D}}\left(c_{\phi_{S}} s_{\psi_{S}} \tilde{\boldsymbol{m}}_{\boldsymbol{y}}+s_{\phi_{S}} s_{\psi_{S}} \tilde{\boldsymbol{m}}_{\boldsymbol{z}}\right) \\
& +S(1-A F) s_{\gamma_{D}}\left(s_{\phi_{S}} s_{\psi_{S}} \tilde{\boldsymbol{m}}_{\boldsymbol{y}}-c_{\phi_{S}} s_{\psi_{S}} \tilde{\boldsymbol{m}}_{\boldsymbol{z}}\right)
\end{aligned}
$$

Equation (13) serves as the measurement model for this filter and those described in subsequent sections. The state vector estimated by this filter is given by

$$
\overrightarrow{\boldsymbol{x}}_{D}=\left\{\begin{array}{c}
\boldsymbol{a}_{0} \\
\boldsymbol{a}_{1} \\
\boldsymbol{a}_{2} \\
\boldsymbol{a}_{3} \\
w_{0} \\
w_{1} \\
w_{2} \\
z_{0} \\
z_{1} \\
z_{2}
\end{array}\right\}
$$

It is assumed that all fit parameters do not vary with time, and thus the state and covariance propagation equations are given by

$$
\begin{gathered}
\dot{\overrightarrow{\boldsymbol{x}}}_{\boldsymbol{D}}=0 \\
\dot{\boldsymbol{P}}_{\boldsymbol{D}}=\boldsymbol{Q}_{\boldsymbol{D}}
\end{gathered}
$$

where $\boldsymbol{P}_{\boldsymbol{D}}$ is the state covariance matrix and $\boldsymbol{Q}_{\boldsymbol{D}}$ is a gain matrix corresponding to the estimated process model error covariance. The measurements used by the filter are the three magnetometer outputs corresponding to Equation (13), and thus the state update is given by

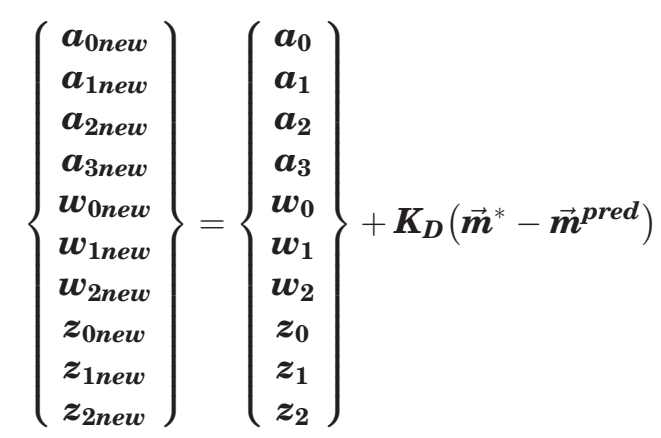

where $\overrightarrow{\boldsymbol{m}}^{*}$ and $\overrightarrow{\boldsymbol{m}}^{\text {pred }}$ are the actual and predicted magnetometer outputs respectively, and $\boldsymbol{K}_{\boldsymbol{D}}$ is the Kalman gain matrix given by

$$
\boldsymbol{K}_{\boldsymbol{D}}=\boldsymbol{P}_{\boldsymbol{D}}\left(\boldsymbol{I}-\boldsymbol{C}_{\boldsymbol{D}}^{T}\left(\boldsymbol{R}_{\boldsymbol{D}}+\boldsymbol{C}_{\boldsymbol{D}} \boldsymbol{P}_{\boldsymbol{D}} \boldsymbol{C}_{\boldsymbol{D}}^{T}\right)^{-1} \boldsymbol{C}_{\boldsymbol{D}} \boldsymbol{P}_{\boldsymbol{D}}\right) \boldsymbol{C}_{\boldsymbol{D}}^{T} \boldsymbol{R}_{\boldsymbol{D}}^{-1}
$$

Note that in Equation (18), $C_{D}$ is the linearized output matrix given by

$\boldsymbol{C}_{\boldsymbol{D}}=\left[\frac{\partial \overrightarrow{\boldsymbol{m}}^{*}}{\partial \boldsymbol{a}_{\mathbf{0}}} \frac{\partial \overrightarrow{\boldsymbol{m}}^{*}}{\partial \boldsymbol{a}_{\mathbf{1}}} \frac{\partial \overrightarrow{\boldsymbol{m}}^{*}}{\partial \boldsymbol{a}_{\mathbf{2}}} \frac{\partial \overrightarrow{\boldsymbol{m}}^{*}}{\partial \boldsymbol{a}_{\mathbf{3}}} \frac{\partial \overrightarrow{\boldsymbol{m}}^{*}}{\partial \boldsymbol{w}_{\mathbf{0}}} \frac{\partial \overrightarrow{\boldsymbol{m}}^{*}}{\partial \boldsymbol{w}_{\mathbf{1}}} \frac{\partial \overrightarrow{\boldsymbol{m}}^{*}}{\partial \boldsymbol{w}_{\mathbf{2}}} \frac{\partial \overrightarrow{\boldsymbol{m}}^{*}}{\partial \boldsymbol{z}_{\mathbf{0}}} \frac{\partial \overrightarrow{\boldsymbol{m}}^{*}}{\partial \boldsymbol{z}_{\mathbf{1}}} \frac{\partial \overrightarrow{\boldsymbol{m}}^{*}}{\partial \boldsymbol{z}_{\mathbf{2}}}\right]$

and $\boldsymbol{R}_{\boldsymbol{D}}$ is a user-specified gain matrix corresponding to the estimated measurement covariance. Finally, state covariance updates are computed according to

$$
\boldsymbol{P}_{\text {DNew }}=\left(\boldsymbol{P}_{D}^{-1}+\boldsymbol{C}_{D}^{\boldsymbol{T}} \boldsymbol{R}_{\boldsymbol{D}}^{-1} \boldsymbol{C}_{\boldsymbol{D}}\right)^{-1}
$$

\section{Magnetometer Nuisance Parameter Estimator}

A separate Extended Kalman filter is used to estimate nuisance parameters for each single-axis magnetometer. The state vector estimated by this filter is given by

$$
\overrightarrow{\boldsymbol{x}}_{\boldsymbol{M}}=\left\{\begin{array}{c}
S \\
b \\
\phi_{S} \\
\psi_{S}
\end{array}\right\}
$$

It is assumed that all nuisance parameters are constant, and thus the state and covariance propagation equations are given by

$$
\begin{gathered}
\dot{\overrightarrow{\boldsymbol{x}}}_{\boldsymbol{M}}=0 \\
\dot{\boldsymbol{P}}_{\boldsymbol{M}}=\boldsymbol{Q}_{\boldsymbol{M}}
\end{gathered}
$$

where $\boldsymbol{P}_{\boldsymbol{M}}$ is the state covariance matrix and $\boldsymbol{Q}_{\boldsymbol{M}}$ is a gain matrix corresponding to the estimated process model error covariance. The sole measurement used by this filter is the magnetometer output $m^{*}$, and thus the state update is given by

$$
\left\{\begin{array}{c}
S_{\text {new }} \\
b_{\text {new }} \\
\phi_{\text {Snew }} \\
\psi_{\text {Snew }}
\end{array}\right\}=\left\{\begin{array}{c}
S \\
b \\
\phi_{S} \\
\psi_{S}
\end{array}\right\}+\boldsymbol{K}_{\boldsymbol{M}}\left(m^{*}-m^{\text {pred }}\right)
$$

In Equation (24), $\boldsymbol{K}_{\boldsymbol{M}}$ is the Kalman gain matrix given by

$$
\boldsymbol{K}_{M}=\boldsymbol{P}_{\boldsymbol{M}}\left(\boldsymbol{I}-\boldsymbol{C}_{\boldsymbol{M}}^{T}\left(\boldsymbol{R}_{M}+\boldsymbol{C}_{\boldsymbol{M}} \boldsymbol{P}_{\boldsymbol{M}} \boldsymbol{C}_{M}^{T}\right)^{-1} \boldsymbol{C}_{\boldsymbol{M}} \boldsymbol{P}_{\boldsymbol{M}}\right) \boldsymbol{C}_{\boldsymbol{M}}^{T} \boldsymbol{R}_{M}^{-1}
$$


where

$$
\boldsymbol{C}_{\boldsymbol{M}}=\left[\begin{array}{llll}
\frac{\partial \boldsymbol{m}^{*}}{\partial \boldsymbol{S}} & \frac{\partial \boldsymbol{m}^{*}}{\partial \boldsymbol{b}} & \frac{\partial \boldsymbol{m}^{*}}{\partial \phi_{\boldsymbol{S}}} & \frac{\partial \boldsymbol{m}^{*}}{\partial \psi_{\boldsymbol{S}}}
\end{array}\right]
$$

and $\boldsymbol{R}_{\boldsymbol{M}}$ is a user-specified gain matrix corresponding to the estimated measurement covariance. Finally, state covariance updates are computed according to

$$
\boldsymbol{P}_{M N e w}=\left(\boldsymbol{P}_{M}^{-1}+\boldsymbol{C}_{M}^{\boldsymbol{T}} \boldsymbol{R}_{M}^{-1} \boldsymbol{C}_{\boldsymbol{M}}\right)^{-1}
$$

Note that three separate instances of this filter (one for each single-axis sensor) actually comprise the nuisance parameter estimator.

\section{Orientation State Estimator}

The orientation estimator uses an EKF framework to combine feedback signals from rate gyros, magnetometers, and the velocity estimator. The process model used by this filter is given by the nonlinear strapdown equations,

$$
\left[\begin{array}{c}
\dot{\hat{\phi}} \\
\dot{\hat{\theta}} \\
\dot{\hat{\psi}}
\end{array}\right]=\left[\begin{array}{ccc}
1 & s_{\hat{\phi}} t_{\hat{\theta}} & c_{\hat{\phi}} t_{\hat{\theta}} \\
0 & c_{\hat{\phi}} & -s_{\hat{\phi}} \\
0 & s_{\hat{\phi}} / c_{\hat{\theta}} & c_{\hat{\phi}} / c_{\hat{\theta}}
\end{array}\right]\left\{\begin{array}{c}
\omega_{x}^{*} \\
\omega_{y}^{*} \\
\omega_{z}^{*}
\end{array}\right\}=[D]\left\{\begin{array}{c}
\omega_{x}^{*} \\
\omega_{y}^{*} \\
\omega_{z}^{*}
\end{array}\right\}
$$

Note that in the above equations, $\hat{\phi}, \hat{\theta}, \hat{\psi}$ are the estimated Euler angles and $\omega_{x}^{*}, \omega_{y}^{*}, \omega_{z}^{*}$ are gyro measurements. As mentioned before, signals from the rate gyroscope are used directly to drive Euler angle dynamics and thus are not used for measurement updates. The Jacobian of $[D]$, omitted here for brevity, is used to propagate the state covariance matrix $\boldsymbol{P}_{\boldsymbol{E}}$ according to

$$
\dot{\boldsymbol{P}}_{\boldsymbol{E}}=\boldsymbol{A}_{\boldsymbol{E}} \boldsymbol{P}_{\boldsymbol{E}}+\boldsymbol{P}_{\boldsymbol{E}} \boldsymbol{A}_{\boldsymbol{E}}^{T}+\boldsymbol{Q}_{\boldsymbol{E}}
$$

where $\boldsymbol{A}_{\boldsymbol{E}}$ is the Jacobian of $[D]$ and $\boldsymbol{Q}_{\boldsymbol{E}}$ a userspecified gain matrix. The measurement vector, given by

$$
M_{E}=\left\{\begin{array}{c}
m_{1}^{*} \\
m_{2}^{*} \\
m_{3}^{*} \\
\theta_{m} \\
\psi_{m}
\end{array}\right\}
$$

is used to update the estimated state vector at a rate of $100 \mathrm{~Hz}$. State and covariance updates are performed by first computing the Kalman gain matrix $\boldsymbol{K}_{\boldsymbol{E}}$ according to

$$
\boldsymbol{K}_{\boldsymbol{E}}=\boldsymbol{P}_{\boldsymbol{E}}\left(\boldsymbol{I}-\boldsymbol{C}_{\boldsymbol{E}}^{T}\left(\boldsymbol{R}_{\boldsymbol{E}}+\boldsymbol{C}_{\boldsymbol{E}} \boldsymbol{P}_{\boldsymbol{E}} \boldsymbol{C}_{\boldsymbol{E}}^{T}\right)^{-1} \boldsymbol{C}_{\boldsymbol{E}} \boldsymbol{P}_{\boldsymbol{E}}\right) \boldsymbol{C}_{\boldsymbol{E}}^{T} \boldsymbol{R}_{\boldsymbol{E}}^{-1}
$$

where $\boldsymbol{C}_{\boldsymbol{E}}$ is the linearized output matrix of the system computed from Equations (11) and (12) and $\boldsymbol{R}_{\boldsymbol{E}}$ is a user-specified gain matrix. Then, the state vector is updated according to

$$
\left\{\begin{array}{c}
\phi_{\text {new }}^{*} \\
\theta_{\text {new }}^{*} \\
\psi_{\text {new }}^{*}
\end{array}\right\}=\left\{\begin{array}{c}
\phi^{*} \\
\theta^{*} \\
\psi^{*}
\end{array}\right\}+\boldsymbol{K}_{\boldsymbol{E}}\left\{\begin{array}{c}
m_{1}^{*}-m_{1}^{\text {pred }} \\
m_{2}^{*}-m_{2}^{\text {pred }} \\
m_{3}^{*}-m_{3}^{\text {pred }} \\
\theta_{m}-\theta^{*} \\
\psi_{m}-\psi^{*}
\end{array}\right\}
$$

where the superscript (pred) indicates predicted measurements. The state covariance matrix is updated according to

$$
\boldsymbol{P}_{E N e w}=\left(\boldsymbol{P}_{E}^{-1}+\boldsymbol{C}_{E}^{\boldsymbol{T}} \boldsymbol{R}_{E}^{-1} \boldsymbol{C}_{E}\right)^{-1}
$$

\section{Observability Analysis}

The distinguishability of each parameter in the estimation problem can be analyzed by considering a state vector consisting of $\phi, \theta, \psi, S, b, \phi_{S}, \psi_{S}, A F$, $\gamma_{D}$, and $\zeta_{D}$. Three of these states can be identified immediately as indistinguishable due to the identical way in which they affect sensor outputs: $\phi, \phi_{S}$, and $\gamma_{D}$. As the projectile rolls, the magnetometer sensor cannot distinguish between a change in roll angle, a change in magnetometer roll angle alignment, and a change in magnetic field distortion angle. This is because the magnetic field is the sole truth source of the estimator, and other sources of roll angle feedback would be required to distinguish between these factors. Therefore, since a primary goal of the system is to provide an estimate of roll angle, $\phi_{S}$ and $\gamma_{D}$ are eliminated from the estimation problem and magnetometer roll alignment angles and the model for distortion angle must be held constant throughout flight.

The distinguishable nature of each variable in the estimation problem can be verified mathematically by examining the magnetometer measurement equation shown in Equation (13). Consider the observability map of the nonlinear system [21] which can be used to determine distinguishability of one parameter with respect to another. For instance, to compute whether $\phi$ is distinguishable with respect to $\phi_{S}$, the observability map would be computed according to

$$
H=\left[\begin{array}{cc}
\frac{\partial m^{*}}{\partial \phi} & \frac{\partial m^{*}}{\partial \phi_{S}} \\
\frac{\partial \dot{m}^{*}}{\partial \phi} & \frac{\partial \dot{m}^{*}}{\partial \phi_{S}}
\end{array}\right]
$$

If $H$ is always singular, then $\phi$ is indistinguishable from $\phi_{S}$ everywhere in the state space. By computing $H$ for each pair, indistinguishable combinations of the state vector can be identified. 


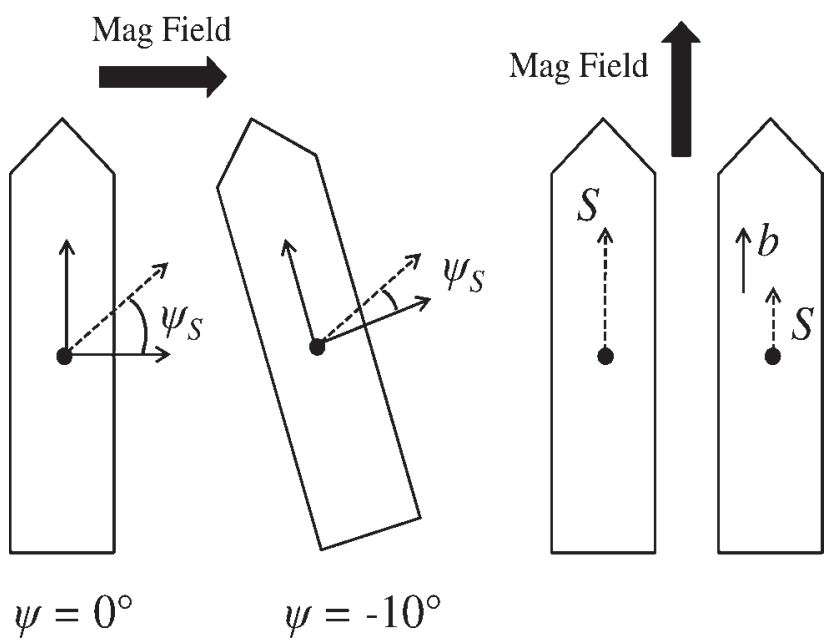

Fig. 8-Unobservable geometries

The observability map was computed for each pair of variables and it was verified that $\phi, \phi_{S}$, and $\gamma_{D}$ are indistinguishable from one another. All other combinations proved to be distinguishable. For nonlinear systems, the statement that two variables are distinguishable in general does not guarantee local observability with respect to one another. In other words, while two parameters may be observable in general, it may be impossible to distinguish between them in certain vehicle orientations. For example, while scale factor, bias, $\psi$, and $\psi_{S}$ are in general observable with respect to one another, there are numerous geometries in which combinations of these quantities are indistinguishable. Figure 8 demonstrates two such geometries (dotted vector represents $\vec{s}$ ). In the leftmost diagram, two different combinations of $\psi$ and $\psi_{S}$ will produce the same magnetometer output in the given field. In the rightmost diagram, two different combinations of bias and scale factor will produce the same magnetometer output in the given field. There are a large number of similar unobservable geometries experienced by the projectile during a typical trajectory. However, even though in some cases unobservable geometries can be momentarily encountered, the integrated filter continues estimation through these points and can recover accuracy when the geometry improves.

\section{RESULTS}

Example estimation results are shown for a 105 mm-diameter fin-stabilized projectile with mass, axial inertia, and transverse inertia given respectively by $17.606 \mathrm{~kg}, 0.0377 \mathrm{~kg}-\mathrm{m}^{2}$, and $0.8530 \mathrm{~kg}$ $\mathrm{m}^{2}$. Two example cases are presented to demonstrate estimator performance. The first case exemplifies the need to incorporate distortion effects by comparing estimation performance between the observer developed here and one that does not compensate for magnetic field distortion. The second case demonstrates performance in a real-world scenario including all uncertainty parameters. All cases in this section are based on the same nominal trajectory in which the projectile is launched with a quadrant elevation of $28.7 \mathrm{deg}$, muzzle velocity of $860 \mathrm{~m} / \mathrm{s}$, and roll rate of $5 \mathrm{rad} / \mathrm{s}$. For the second example, to examine the effects of control inputs on the projectile, $67 \mathrm{~N}$ control pulses of $0.1 \mathrm{sec}$ duration are exerted after apogee in alternating opposite directions in a no-roll frame every second, resulting in angle of attack perturbations but minimal trajectory alteration. These control pulses are added so as to create realistic control-induced angle of attack perturbations that reduce the accuracy of inertial velocity-based orientation estimates. Without the addition of these pulses, the projectile would fly a very smooth ballistic flight path and inertial velocities could be used by themselves to determine pitch and yaw with extreme accuracy. Such a trajectory is not representative of smart weapons flight profiles.

Figures 9-12 show trajectory time histories for both nominal cases (in which control pulses are and are not incorporated). In Figure 10, deflection occurs without control inputs due to gravitational interaction with projectile spin. In Figure 11, control inputs result in pitch and yaw angle perturbations on the order of a few degrees. The no-control case is overlaid with the controlled example, except without the pitch and yaw angle perturbations.

Throughout this section, it is assumed that the projectile body has similar dimensions to the cylinder described in the second section of the paper (neglecting the nose cone and fins) and thus similar distortion characteristics. Furthermore, it is assumed that the magnetometer is placed in the selected example location as also described in the

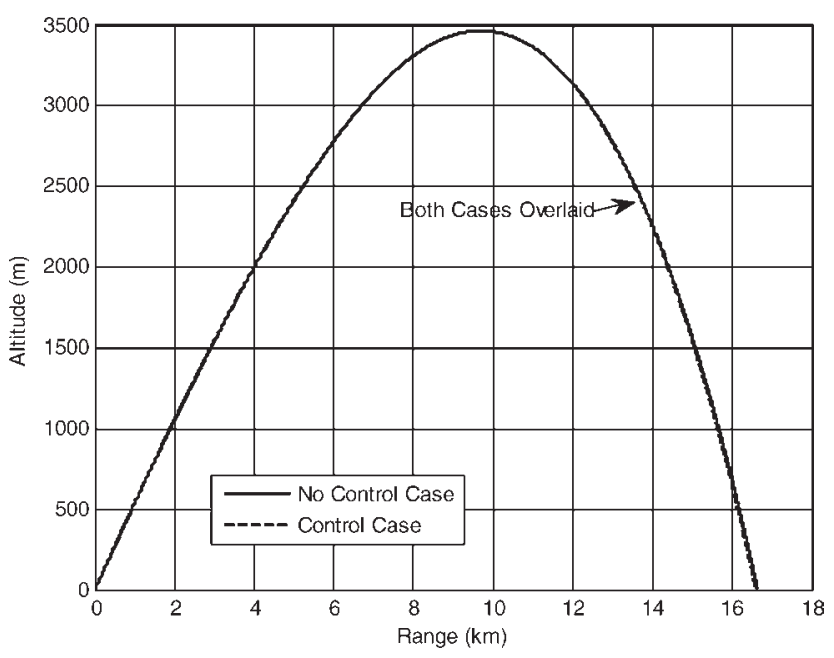

Fig. 9-Altitude vs range for both nominal trajectories 


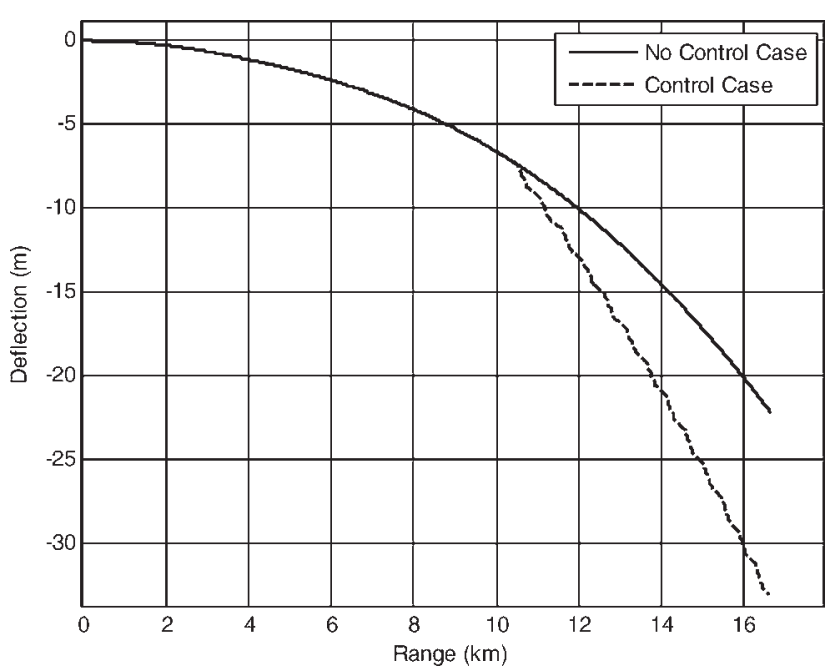

Fig. 10-Deflection vs range for nominal trajectories

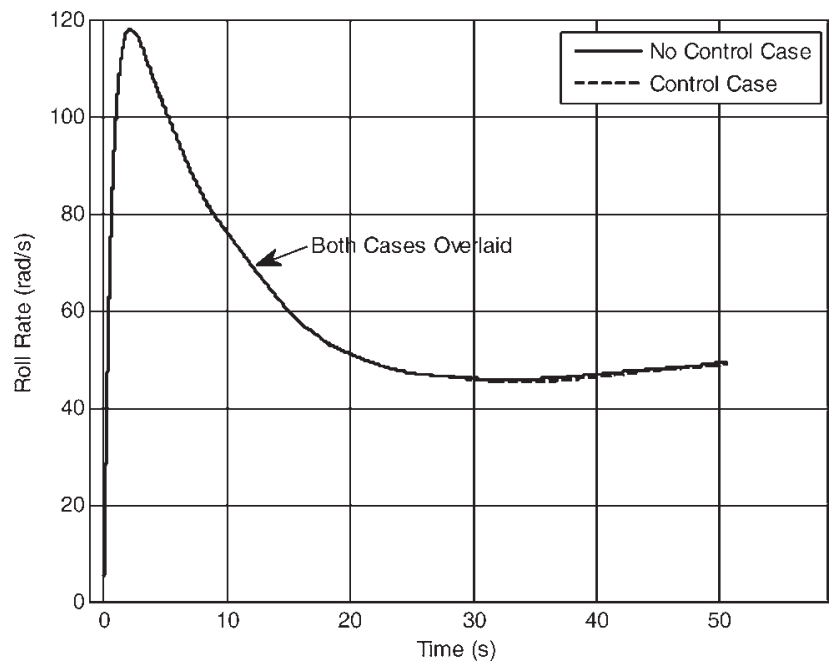

Fig. 11-Projectile roll rate vs time for nominal trajectories

second section. Performance of the integrated attitude-nuisance parameter estimator is examined by considering both example and Monte Carlo simulation results. The following section outlines two sets of example results, while the subsequent section describes Monte Carlo simulation results.

\section{Example Results}

Three primary feedback sources are assumed to provide measurement data to the integrated estimator: a three-axis magnetometer aligned with the projectile body axes (modeled as three single-axis magnetometers), a three-axis gyroscope aligned with the body axes, and an inertial velocity estimator. Feedback errors associated with each measurement source are listed in Table 1, as are their randomly-generated values for the second example
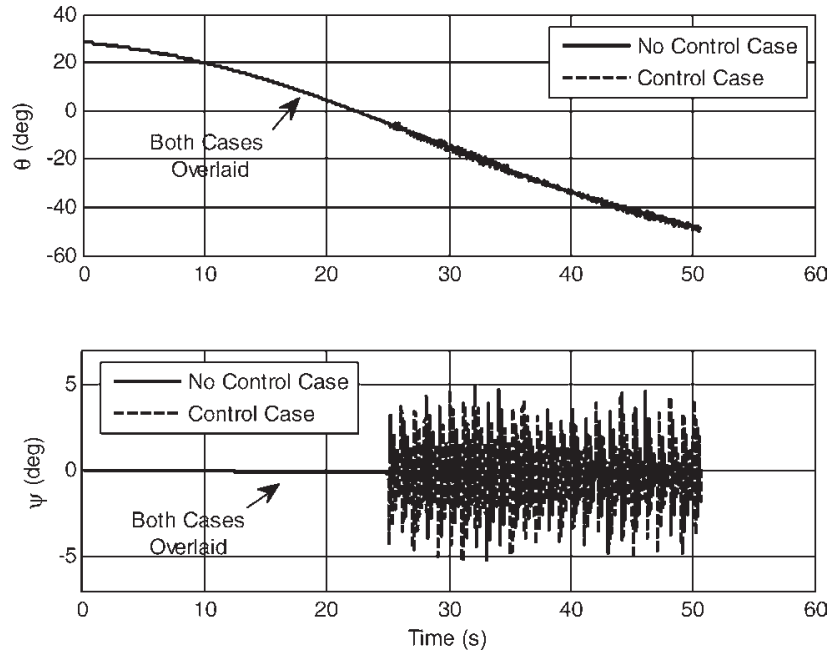

Fig. 12-Pitch and yaw angle vs time for example trajectories

case and standard deviations used for the Monte Carlo case in the next section. In addition, magnetic field distortion fit parameters are listed in Table 2. Note that for the Monte Carlo simulation, only $a_{0}, w_{0}$, and $z_{0}$ were varied indicating that actual distortion curves are likely to have the same general shape as the nominal model obtained from calibration, with uncertainty taking the form of a reasonable additive offset.

Realistic values for the Earth's magnetic field are generated for all cases using the World Magnetic Model, an industry-standard code produced jointly by the US National Geospatial-Intelligence Agency and the UK's Defence Geographic Centre [22]. For the Monte Carlo cases, magnetic field vectors were generated at random from locations between $-45^{\circ}$ and $+45^{\circ}$ latitude. Uncertainty in the magnetic field is also included in the second example simulation and is described in Table 1. Note also that normalized units are used for all magnetic field calculations, obtained by normalizing all components by the magnitude of the Earth's field for that particular case.

Two example cases are shown in order to characterize the effects of nuisance parameters and spininduced field distortion as well as overall performance of the integrated estimator. The first case examines the effect of field distortion by considering two orientation estimators: one using a magnetometer model that neglects spin-induced distortion (based on Equation (10)), and the other using the estimator developed here (based on Equation (13)). In order to isolate the effect of field distortion, both estimators are assumed to have perfect knowledge of magnetometer nuisance parameters while the latter estimator is assumed to also have perfect knowledge of field distortion as a function of spin. Furthermore, in both cases, gyro and inertial veloc- 
Table 1-Feedback Error Parameters

\begin{tabular}{|c|c|c|c|c|}
\hline Feedback Source & Description & Parameter & Example Case 2 Value & $\begin{array}{l}\text { Std. Deviation for } \\
\text { MC Simulations }\end{array}$ \\
\hline \multirow[t]{5}{*}{ Magnetometer } & Scale Factor & $S_{1}, S_{2}, S_{3}(\mathrm{nd})$ & $0.998,1.033,0.990$ & 0.03 \\
\hline & Bias & $\begin{array}{l}b_{1}, b_{2}, b_{3} \text { (norm. } \\
\text { units) }\end{array}$ & $0.236,-0.017,-0.212$ & 0.7 Gauss \\
\hline & $\phi_{S}$ Alignment Angle & $\phi_{S 1}, \phi_{S 2}, \phi_{S 3}(\operatorname{deg})$ & $2.48,0.430,91.4$ & 1.0 \\
\hline & $\psi_{S}$ Alignment Angle & $\psi_{S 1}, \psi_{S 2}, \psi_{S 3}(\mathrm{deg})$ & $0.905,89.28,89.88$ & 1.0 \\
\hline & Noise Std. Dev. & $n$ (norm. units) & 0.01 & N/A \\
\hline \multirow[t]{6}{*}{ Gyros } & Scale Factor & $S_{G x}, S_{G y}, S_{G z},(\mathrm{nd})$ & $1.052,0.991,0.993$ & 0.03 \\
\hline & Bias & $b_{p}, b_{q}, b_{r}(\mathrm{rad} / \mathrm{s})$ & $1.529,-0.787,1.007$ & $5.0,1.0,1.0$ \\
\hline & $\omega_{x}$ Cross Axis & $c^{p q}, c^{p r}(\mathrm{nd})$ & $-0.0038,0.0072$ & 0.01 \\
\hline & $\omega_{y}$ Cross Axis & $c^{q p}, c^{q r}(\mathrm{nd})$ & $0.0016,0.0087$ & 0.01 \\
\hline & $\omega_{z}$ Cross Axis & $c^{r p}, c^{r q}(\mathrm{nd})$ & $0.0190,0.0056$ & 0.01 \\
\hline & Noise Std. Dev. & $n_{p}, n_{r}, n_{q}(\mathrm{rad} / \mathrm{s})$ & 0.05 & N/A \\
\hline \multirow[t]{2}{*}{ Velocity Estimator } & $\operatorname{Bias}(\mathrm{m} / \mathrm{s})$ & $b_{v x}, b_{v y}, b_{v z},(\mathrm{~m} / \mathrm{s})$ & $0.95,-0.60,3.40$ & $1.52,1.52,3.40$ \\
\hline & Noise Std. Dev. & $n_{v}(\mathrm{~m} / \mathrm{s})$ & 3.05 & N/A \\
\hline \multirow[t]{3}{*}{$\begin{array}{l}\text { Earth's Magnetic } \\
\text { Field }\end{array}$} & $\begin{array}{l}\text { Field } N, E, D \\
\quad \text { components }\end{array}$ & $\begin{array}{l}m_{N}, m_{E}, m_{D} \text { (norm. } \\
\quad \text { units) }\end{array}$ & $0.362,-0.054,-0.930$ & N/A \\
\hline & $\begin{array}{l}\text { Errors in Knowledge } \\
\text { Magnitude of Field }\end{array}$ & $\begin{array}{l}\Delta m_{\text {mag }}(\text { norm. } \\
\text { units })\end{array}$ & -0.045 & 0.03 \\
\hline & $\begin{array}{l}\text { Errors in Knowledge } \\
\text { of Az. and El. of } \\
\text { Field }\end{array}$ & $\Delta m_{a z}, \Delta m_{e l}(\mathrm{deg})$ & $-0.246,-0.120$ & 0.2 \\
\hline
\end{tabular}

Table 2-Magnetic Field Distortion Curve Fit Parameters

\begin{tabular}{|c|c|c|c|c|c|}
\hline Parameter & Value & Parameter & Value & Parameter & Value \\
\hline$a_{0}$ & $-3.11 \times 10^{-3}$ & $w_{0}$ & $-1.43 \times 10^{-2}$ & $z_{0}$ & $-1.58 \times 10^{-3}$ \\
\hline$a_{1}$ & $8.12 \times 10^{-5}$ & $w_{1}$ & $8.04 \times 10^{-3}$ & $z_{1}$ & $-5.13 \times 10^{-4}$ \\
\hline$a_{2}$ & $2.46 \times 10^{-5}$ & $w_{2}$ & $-1.42 \times 10^{-5}$ & $z_{2}$ & $3.46 \times 10^{-6}$ \\
\hline$a_{3}$ & $-7.99 \times 10^{-8}$ & & & & \\
\hline $\begin{array}{l}\text { Initial } a_{0} \\
\quad(\text { Example 2) }\end{array}$ & $-1.02 \times 10^{-2}$ & Initial $w_{0}$ (Example 2) & $-3.87 \times 10^{-2}$ & Initial $z_{0}$ (Example 2) & $-3.67 \times 10^{-3}$ \\
\hline $\begin{array}{c}\text { Init. } a_{0} \text { Std. Dev. } \\
\text { (Monte Carlo) }\end{array}$ & $9.34 \times 10^{-3}$ & $\begin{array}{l}\text { Init. } w_{0} \text { Std. Dev. } \\
\text { (Monte Carlo) }\end{array}$ & $4.29 \times 10^{-2}$ & $\begin{array}{r}\text { Init. } z_{0} \text { Std. Dev. } \\
\text { (Monte Carlo) }\end{array}$ & $4.73 \times 10^{-3}$ \\
\hline
\end{tabular}

ity feedback is assumed to be perfect and the Earth's magnetic field is assumed to be known exactly. The purpose of this example case is to demonstrate expected estimation error for magnetometer-based observers that neglect spin-induced distortion. For all example cases in this section, the projectile is launched at an azimuth angle of approximately $43 \mathrm{deg}$ West of North and estimation commences $3 \mathrm{sec}$ into the trajectory at a rate of $100 \mathrm{~Hz}$.

Figures 13 and 14 show roll, pitch, and yaw estimation error exhibited by both estimators for this example case. Figure 13 demonstrates that the estimator that does not account for magnetic field distortion suffers roll estimation errors consistently between 20 and $40 \mathrm{deg}$, while Figure 14 shows that pitch angle estimation error on the order of a few degrees occurs when distortion is not accounted for. The integrated estimator developed here is able to properly account for all distortion effects and thus demonstrates estimation error near zero for all angles. Note that this example case represents an ideal scenario in which all nuisance parameters were assumed to be zero and other sensor feedback was assumed to be perfect. In a realistic environment, roll angle estimation error for an observer that does not account for distortion would likely be even worse than demonstrated here. Furthermore, note that typical magnetometer nuisance parameters (scale factor, bias, and misalignment) cannot properly compensate for magnetic field distortion effects since these appear largely as a transverse 


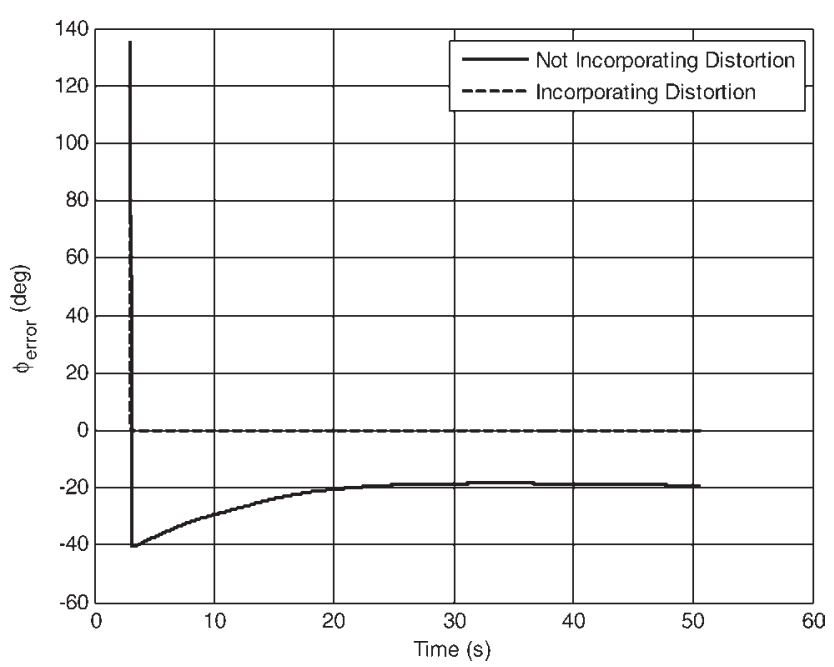

Fig. 13-Roll estimation error vs time for example case 1
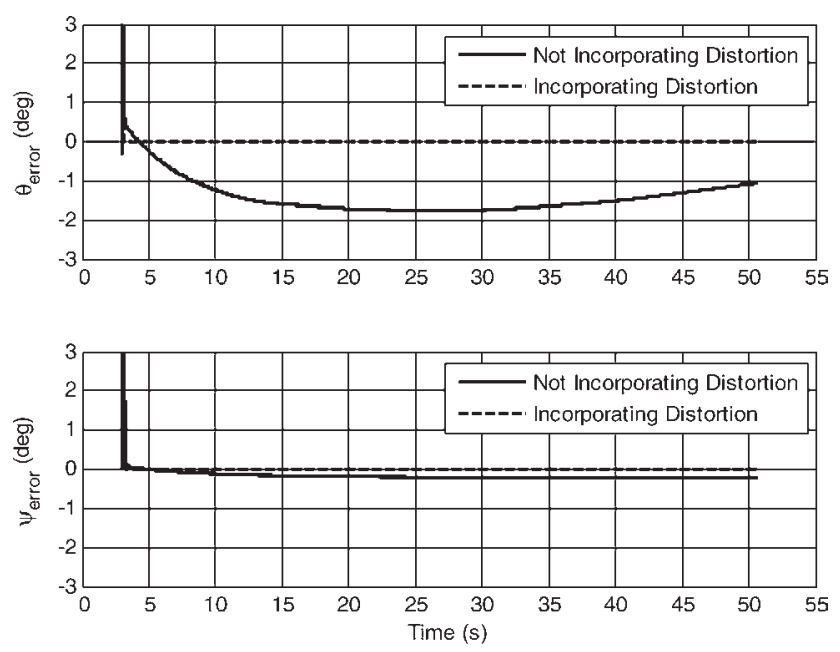

Fig. 14-Pitch and yaw estimation errors vs time for example case 1

distortion angle (i.e., $\gamma_{D}$ is the dominant effect). The only parameter that could account for such an effect is the magnetometer roll alignment angle, $\phi_{S}$, which was shown to be unobservable and thus removed from the estimation problem. The example results shown here demonstrate that incorporation of spin-induced distortion effects is critical to ensuring estimation accuracy for magnetometer-based filters in rapidly-spinning flight bodies.

The second example demonstrates estimator performance in the presence of reasonable sensor errors and uncertainty in the Earth's magnetic field. Error and uncertainty parameters for this example case are listed in Tables 1 and 2 . As in the first example, orientation estimation begins at $3 \mathrm{sec}$ into the trajectory using nominal guesses for magnetometer nuisance parameters. Then, at $6 \mathrm{sec}$ both the nuisance parameter estimator and the distortion parameter estimator begin to provide
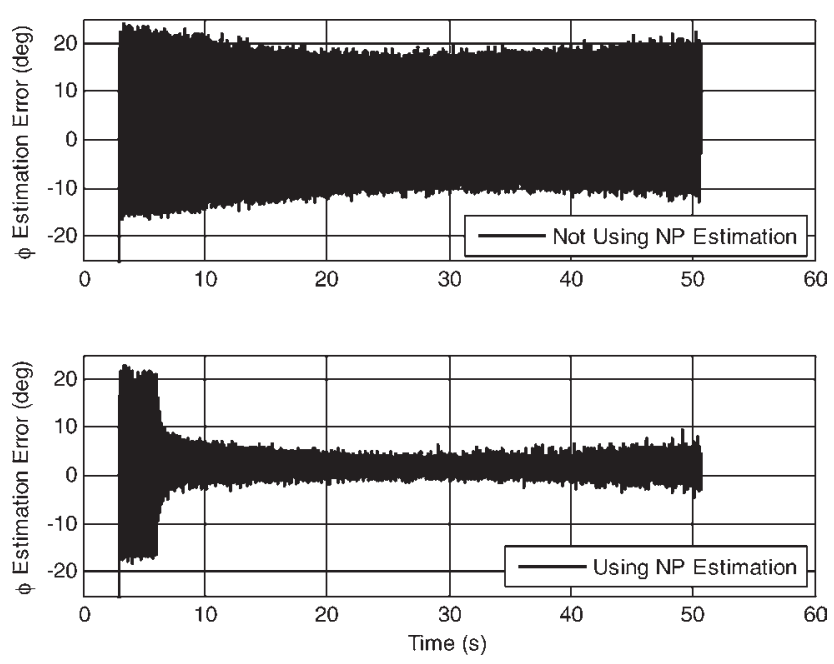

Fig. 15- $\phi$ Estimation error vs time for second example case
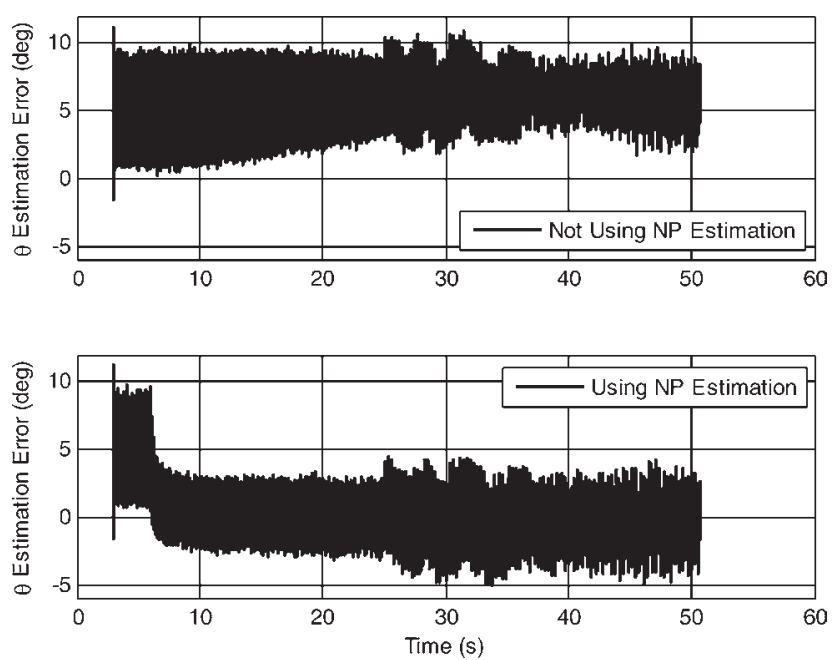

Fig. 16-0 Estimation error vs time for second example case

updates to their respective states at a rate of 100 Hz. In Figures 15-17, this case is referred to as "Using NP Estimation."

This estimation case is compared to a case in which the nuisance parameter and distortion parameter filters are not activated. In this case, orientation estimation begins at $3 \mathrm{sec}$ and nuisance parameters and distortion fit parameters are held constant at their initial values (referred to in Figures 15-22 as "Not Using NP Estimation"). Figures 15-22 show comparisons of filter performance for these two trajectories. Figures 15, 16, and 17 show time histories of $\phi$ estimation error, $\theta$ estimation error, and $\psi$ estimation error, respectively. For the case in which nuisance parameter estimation was used, Figure 18 shows a time history of the difference between measured and predicted magnetometer outputs for each sensor, while Figures 19, 20, and 21 show time histories of scale factor estima- 

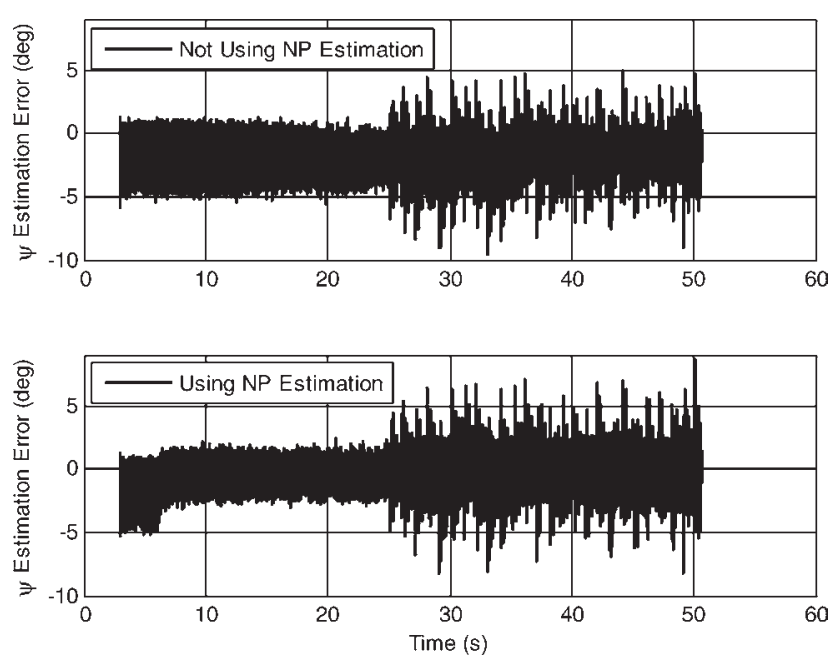

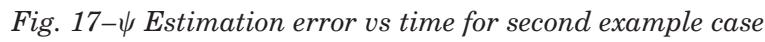

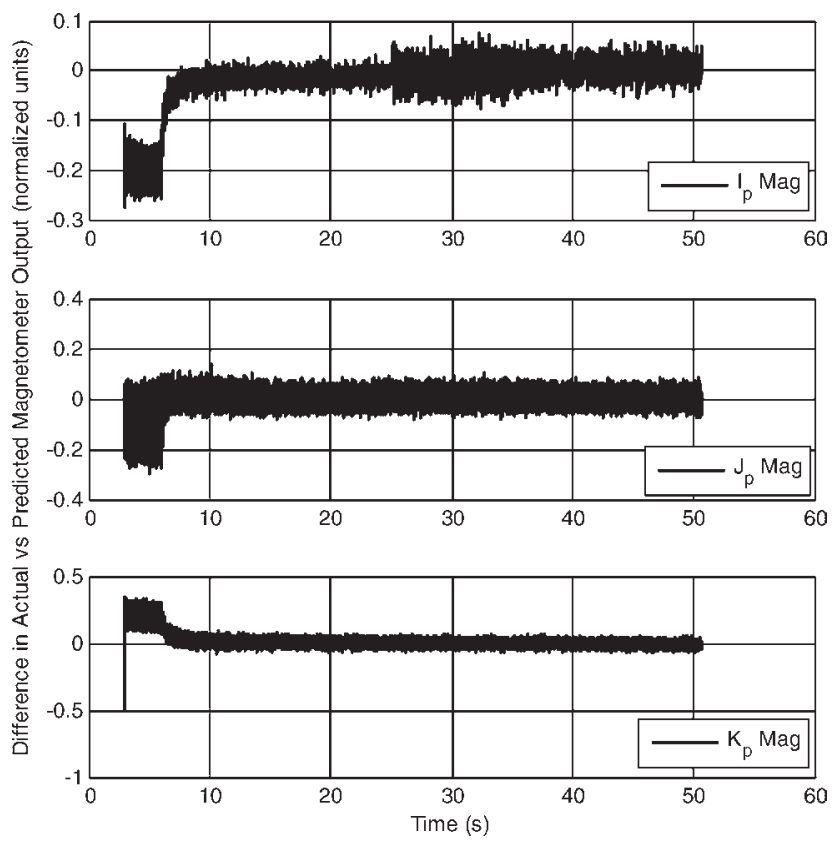

Fig. 18-Difference between measured and predicted magnetometer output for second example case

tion error, bias estimation error, and $\psi_{S}$ alignment angle estimation error for each magnetometer, respectively. In Figures 19-21, the dotted lines represent $1 \sigma$ error covariance estimates output from the Kalman filter. Finally, Figure 22 shows a time history of percent error in $A F$ and $\zeta_{D}$ estimates.

Figures 15-22 demonstrate very interesting, and to a certain extent, unexpected phenomena when nuisance and distortion parameter estimation is performed. First, Figures 15-17 demonstrate that attitude estimation performance is greatly enhanced after nuisance and distortion parameter estimation is activated (after $6 \mathrm{sec}$ ). Specifically, maximum error in $\phi$ is reduced from about $20 \mathrm{deg}$
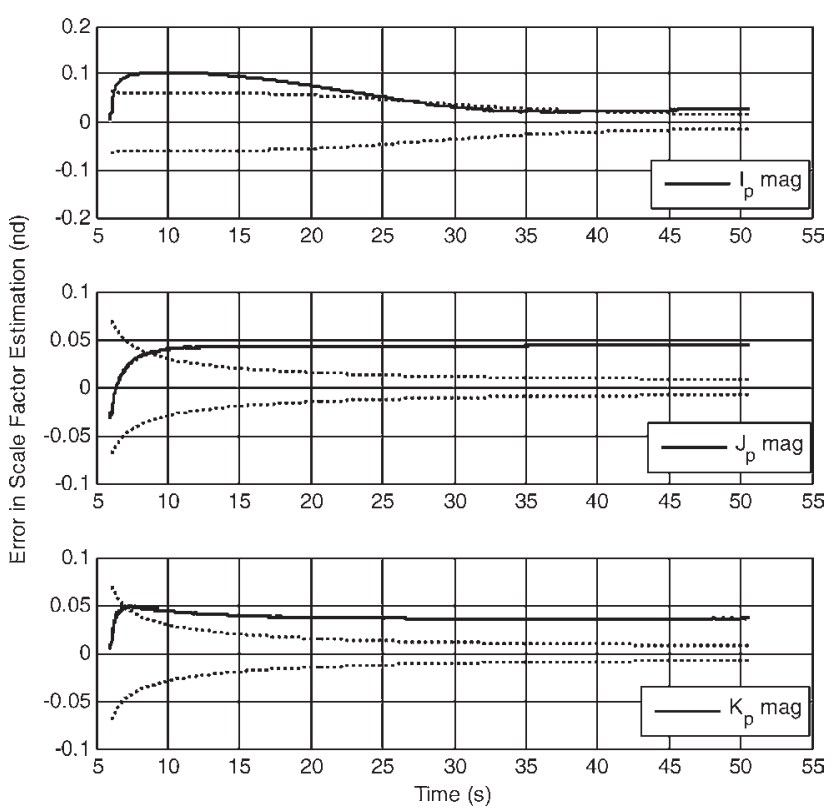

Fig. 19-Scale factor estimation error vs time for second example case

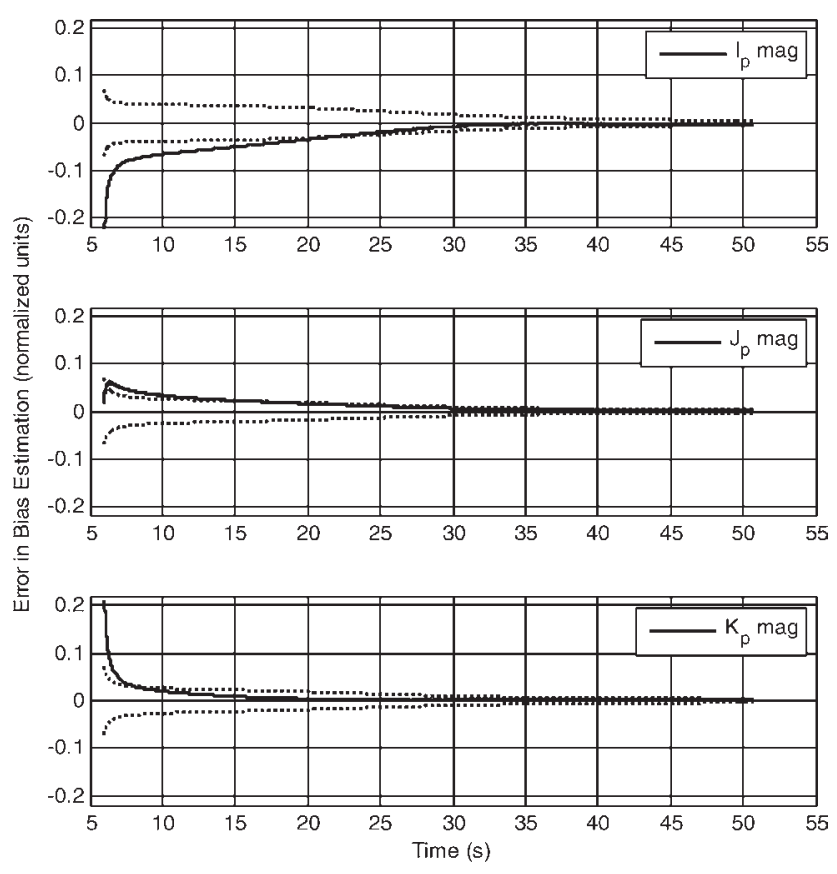

Fig. 20-Bias estimation error vs time for second example case

to less than $7 \mathrm{deg}$, maximum error in $\theta$ is reduced from about 8 deg to less than $4 \mathrm{deg}$, and maximum error in $\psi$ is reduced from about 5 deg to less than $3 \mathrm{deg}$ (before control activation). This error reduction, especially in roll angle estimation, likely would have considerable positive impact on control performance.

Figure 18 demonstrates that, as expected, after nuisance and distortion parameter estimation 

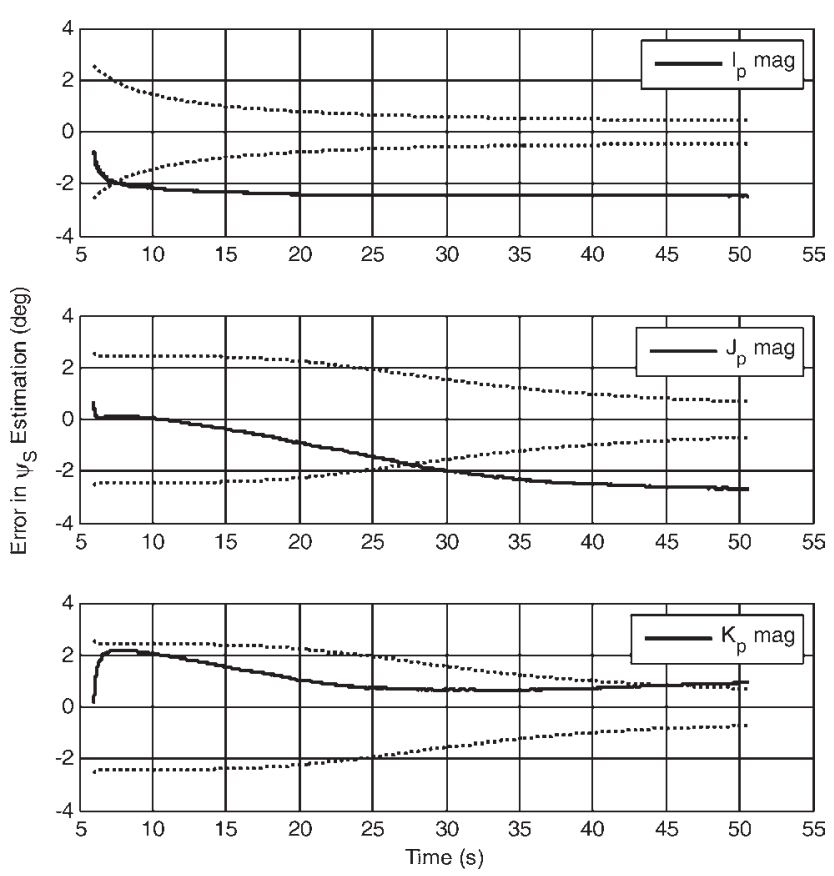

Fig. $21-\psi_{S}$ Estimation error vs time for second example case

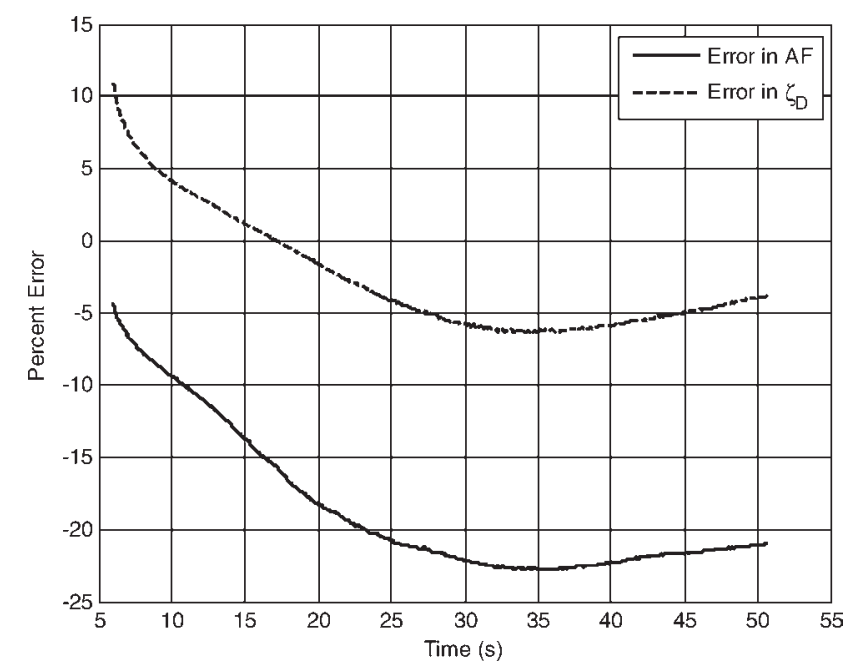

Fig. 22-Percent error in distortion parameters vs time for second example case

begins, the error between measured and predicted magnetometer outputs decreases significantly. This is the result of the nuisance parameter and distortion parameter estimators tuning their respective values so that the measured data matches predicted data. However, while Figure 20 demonstrates that the filter accurately estimated all magnetometer biases, scale factor and $\psi_{S}$ converged to incorrect values for each sensor. This type of behavior was observed throughout many example cases, and is the result of poor local observability conditions between these two parameters. As described in the Observability Analysis Section, although each estimated nuisance parameter is mathematically observable, in many flight scenarios (such as the example shown here) many combinations of nuisance parameters will lead to similar signal outputs. Thus, although nuisance parameter estimates might converge to incorrect values, magnetometer signals may more closely reflect predicted measurements. As shown in Figures 19-21, by the end of the trajectory $1 \sigma$ covariance estimates are small for both scale factor and $\psi_{S}$ because the observed and predicted magnetometer outputs match reasonably well even though some nuisance parameters converged to incorrect values.

Figure 22 shows that error in $A F$ and $\zeta_{D}$ actually grew after the distortion parameter estimator was activated. Again this is the result of poor observability between $A F, \zeta_{D}$, scale factor, and errors in knowledge of the magnetic field (which, in this case, represent errors in a truth source and cannot be compensated for). Thus, errors in knowledge of the magnetic field and magnetometer nuisance parameters can contaminate attenuation factor estimates and again cause convergence to the wrong value.

The most important result demonstrated by this example case is that, even if nuisance parameters and distortion fit parameters converge to incorrect values, attitude estimation performance almost universally improves. This is because, if nuisance and distortion parameters are fixed, the orientation estimator is forced to alter $\phi, \theta$, and $\psi$ when discrepancies are observed between predicted and actual magnetometer outputs. When nuisance and distortion parameter estimation is incorporated, the integrated filter can tune these parameters such that predicted data is closer to measured data, thus removing the burden on the orientation filter. Since the primary goal of the integrated estimator is to obtain estimates for $\phi, \theta$, and $\psi$, nuisance and distortion parameter estimation pays off in spite of the tendency for some of these parameters to display poor observability and converge to incorrect values.

\section{Monte Carlo Simulation}

To demonstrate that orientation estimation performance invariably improves when nuisance and distortion parameter estimation is incorporated, a Monte Carlo simulation is performed. The controlled example trajectory was run 1,000 times, and in each case magnetometer, gyro, and velocity feedback error parameters were perturbed according to the standard deviations shown in Tables 1 and 2 . For each case, the Earth's magnetic field vector was selected at random from a location between $-45^{\circ}$ and $+45^{\circ}$ latitude using the World Magnetic Model, and launch azimuth angle with respect to North was varied uniformly between 0 and $360 \mathrm{deg}$. The 

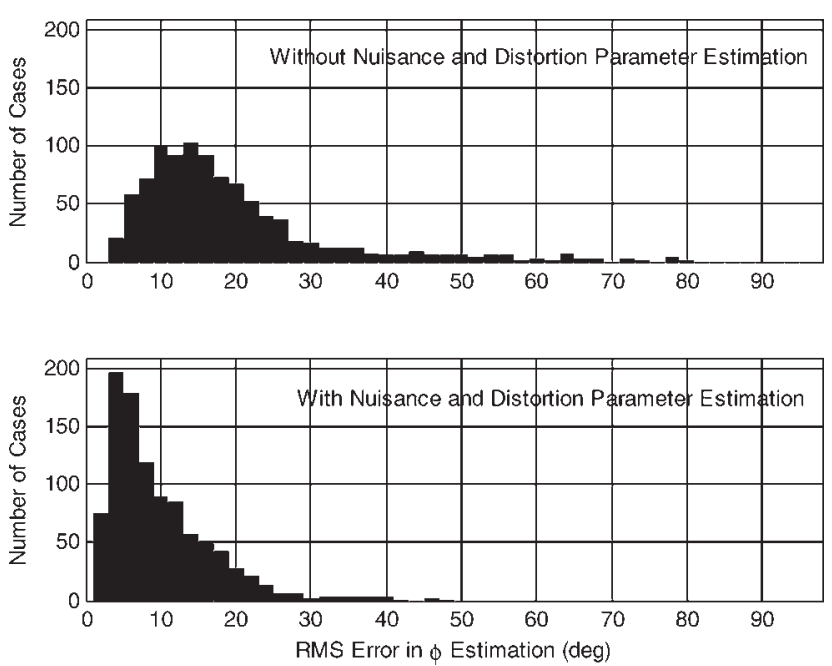

Fig. 23-Histogram of RMS errors in $\phi$ estimation (1,000 total cases)

initial guess for pitch angle was $0.46 \mathrm{rad}$ in each case, while the initial guess for roll angle was varied uniformly between 0 and $2 \pi$ rad. Two cases were run for each set of initial conditions: one case in which nuisance and distortion parameter estimation was performed, and one case in which nuisance and distortion parameter estimation was not. For each trajectory, RMS errors were calculated for $\phi$, $\theta$, and $\psi$ estimation.

Results of the Monte Carlo simulation are shown in Figures 23-25. These figures show histograms of RMS estimation error for $\phi, \theta$, and $\psi$, respectively, for the cases in which nuisance and distortion parameter estimation is and is not included in the estimation process. All figures clearly demonstrate that the filter that incorporates nuisance and dis-
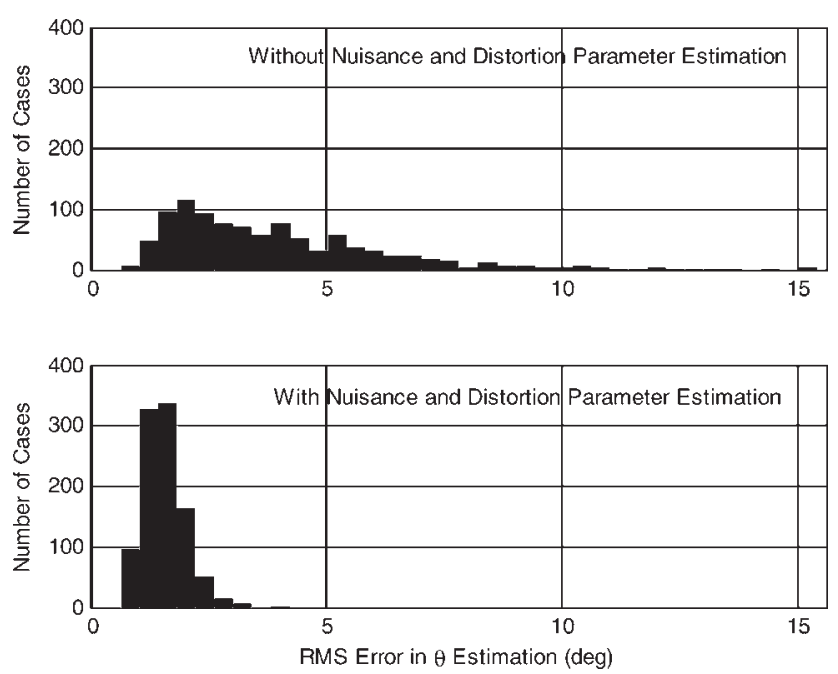

Fig. 24-Histogram of RMS errors in $\theta$ estimation (1,000 total cases)
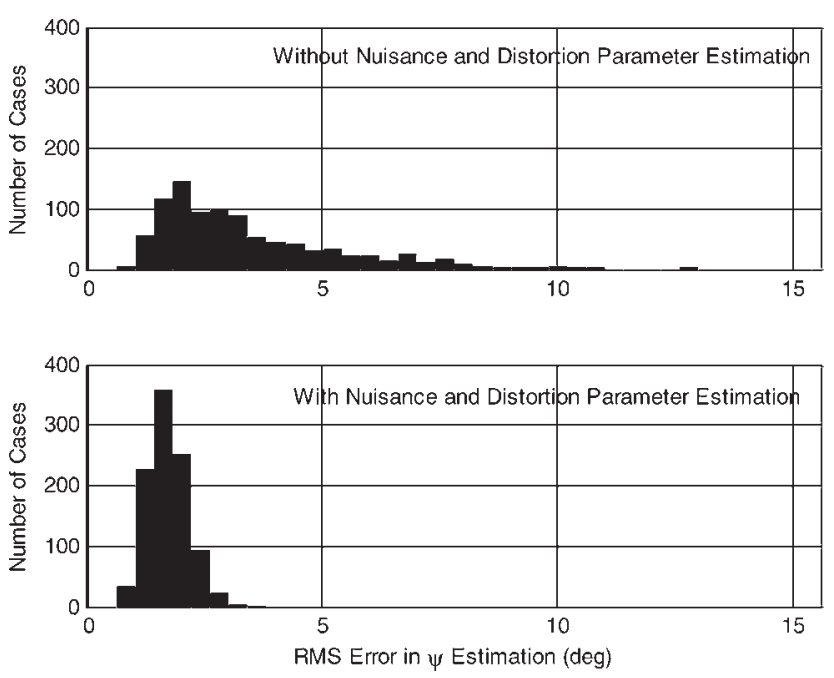

Fig. 25-Histogram of RMS errors in $\psi$ estimation (1,000 total cases)

tortion parameter estimation performs better than the filter without it.

Tables 3 and 4 provide a statistical summary of the Monte Carlo results ("No NP" denotes that no nuisance and distortion parameter estimation was used; "NP" denotes that nuisance and distortion parameter estimation was used). Note that, for estimation of each Euler angle, all statistical measures show significant improvement when nuisance and distortion parameter estimation is incorporated. In Table 3, mean RMS errors for all angles show greater than $50 \%$ reduction and error variances for all angles show greater than $75 \%$ reduction when nuisance and distortion parameter estimation is performed. The confidence intervals shown in Table 4 also clearly demonstrate that estimation of all angles improves considerably when parameter estimation is incorporated. However, note that in most cases nuisance parameter convergence to incorrect values occurred to some extent due to poor observability scenarios. Thus the primary result demonstrated by this Monte Carlo example is that, in general, online magnetometer calibration results in improved estimation even though convergence of parameters to their true values is not guaranteed, since the filter can tune alternative parameters in the measurement equation without having to disrupt Euler angle estimates.

Table 3-Mean and Variance of Monte Carlo Results

\begin{tabular}{r|rcc|rcc}
\hline & \multicolumn{3}{|c|}{ Mean (deg) } & \multicolumn{3}{c}{ Variance $\left(\mathrm{deg}^{2}\right)$} \\
\hline & No NP & NP & \% Reduction & No NP & NP & \% Reduction \\
\hline$\phi$ & 20.26 & 9.89 & 51.2 & 220.8 & 50.7 & 77.0 \\
$\theta$ & 3.97 & 1.54 & 61.2 & 5.80 & 0.21 & 96.4 \\
$\psi$ & 3.58 & 1.70 & 52.5 & 5.06 & 0.19 & 96.3 \\
\hline
\end{tabular}


Table 4-Confidence Intervals of Monte Carlo Results (Results in Degrees)

\begin{tabular}{|c|c|c|c|c|c|c|c|c|c|}
\hline & \multicolumn{3}{|c|}{$50 \%$ Confidence Interval } & \multicolumn{3}{|c|}{$75 \%$ Confidence Interval } & \multicolumn{3}{|c|}{$90 \%$ Confidence Interval } \\
\hline & No NP & NP & $\%$ Red. & No NP & NP & $\%$ Red. & No NP & NP & $\%$ Red \\
\hline$\phi$ & 16.06 & 7.81 & 51.4 & 23.51 & 13.11 & 44.2 & 38.92 & 19.00 & 51.2 \\
\hline$\theta$ & 3.34 & 1.48 & 55.7 & 5.17 & 1.79 & 65.4 & 7.04 & 2.13 & 69.7 \\
\hline$\psi$ & 2.88 & 1.66 & 42.4 & 4.52 & 1.96 & 46.4 & 6.70 & 2.25 & 66.4 \\
\hline
\end{tabular}

\section{CONCLUSION}

The use of magnetometers for orientation estimation on-board projectiles is considered. In particular, the effect of spin-induced magnetic field distortion is discussed. Analytical, finite element, and experimental techniques are used to characterize magnetic field distortion effects for typical projectile configurations. Then, an estimation algorithm is developed that demonstrates how compensation for field distortion effects can be performed. The estimation algorithm is responsible for orientation estimation as well as distortion and magnetometer nuisance parameter estimation, and consists of three coupled Extended Kalman filters. An analytical observability analysis is then performed to identify globally indistinguishable states, which are then removed from the estimation problem. An example simulation demonstrates that standard magnetometerbased observers that do not compensate for spininduced distortion are likely to suffer significant estimation error. Finally, a Monte Carlo simulation directly compares estimators with and without nuisance and distortion parameter estimation capability, and it is shown that parameter estimation enables significant attitude estimator performance increases.

\section{REFERENCES}

1. Wahba, G., "Problem 65-1: A Least Squares Estimate of Satellite Attitude," SIAM Review, Vol. 7, No. 3, July 1965, p. 409.

2. Wahba, G., et al., "Problem 65-1 (Solution)," SIAM Review, Vol. 8, August 1966, pp. 384-386.

3. Shuster, M. D. and Oh, S. D., "Three-Axis Attitude Determination from Vector Observations," Journal of Guidance and Control, Vol. 4, No. 1, Jan.-Feb. 1981, pp. 70-77.

4. Bar-Itzhak, I. Y. and Reiner, J., "Recursive Attitude Determination from Vector Observations: Direction Cosine Matrix Identification," Journal of Guidance, Control, and Dynamics, Vol. 7, No. 1, Jan.-Feb. 1984, pp. 51-56.

5. Bar-Itzhak, I. Y. and Oshman, Y., "Attitude Determination from Vector Observations: Quaternion Estimation," IEEE Transactions on Aerospace and Electronic Systems, Vol. AES-21, No. 1, January 1985, pp. 128136.
6. Bar-Itzhak, I. Y. and Idan, M., "Recursive Attitude Determination from Vector Observations: Euler Angle Estimation," Journal of Guidance, Control, and Dynamics, Vol. 10, No. 2, March-April 1987, pp. 152 157.

7. Mortari, D., "Euler-q Algorithm for Attitude Determination from Vector Observations," Journal of Guidance, Control, and Dynamics, Vol. 21, No. 2, MarchApril 1998, pp. 328-334.

8. Psiaki, M. L., "Global Magnetometer-Based Spacecraft Attitude and Rate Estimation," Journal of Guidance, Control, and Dynamics, Vol. 27, No. 2, MarchApril 2004, pp. 240-250.

9. Ohlmeyer, E., Fraysse, J., and Pepitone, T. R., "Guidance, Navigation, and Control Without Gyros: A Gun-Launched Munition Concept," AIAA Guidance, Navigation, and Control Exhibit, Monterey, CA, August 5-8, 2002, Paper AIAA-2002-5025.

10. Wilson, M. J., "Onboard Attitude Determination for Gun-Launched Projectiles," $43^{\text {rd }}$ AIAA Aerospace Sciences Meeting and Exhibit, Reno, NV, January 1013, 2005, Paper AIAA-2005-1217.

11. Lee, H., Kim, K., and Park, H., "Roll Estimation of a Smart Munition Using a Magnetometer Based on an Unscented Kalman Filter," AIAA Guidance, Navigation, and Control Exhibit, Honolulu, HI, August 1821, 2008, Paper AIAA-2008-7460.

12. Harkins, T., "On the Viability of Magnetometer-Based Projectile Orientation Measurements," US Army Research Laboratory Technical Report ARL-TR-4310, November 2007.

13. Lerner, G. M. and Shuster, M. D., "In-Flight Magnetometer Calibration and Attitude Determination for Near-Earth Spacecraft," Journal of Guidance and Control, Vol. 4, No. 5, September-October 1981, pp. 518-522.

14. Alonso, R. and Shuster, M. D., "Complete Linear Attitude-Independent Magnetometer Calibration," Journal of the Astronautical Sciences, Vol. 50, No. 4, October-December 2002, pp. 477-490.

15. Alonso, R. and Shuster, M. D., "Centering and Observability in Attitude-Independent Magnetometer-Bias Determination," Journal of the Astronautical Sciences, Vol. 51, No. 2, April-June 2003, pp. 133-141.

16. Alonso, R. and Shuster, M. D., "Attitude-Independent Magnetometer-Bias Determination - A Survey," Journal of the Astronautical Sciences, Vol. 50, No. 4, October-December 2002, pp. 453-475.

17. Crassidis, J. L., Lai, K., and Harman, R. R., "Real-Time Attitude-Independent Three-Axis Magnetometer Calibration," Journal of Guidance, Control, and Dynamics, Vol. 28, No. 1, January-February 2005, pp. 115-120. 
18. Gebre-Egziabher, D., "Magnetometer Autocalibration Leveraging Measurement Locus Constraints," Journal of Aircraft, Vol. 44, No. 4, July-August 2007, pp. 1361-1368.

19. Perry, M. P. and Jones, T. B., "Eddy Current Induction in a Solid Conducting Cylinder with a Transverse Magnetic Field," IEEE Transactions on Magnetics, Vol. MAG-14, No. 4, July 1978, pp. 227-232.

20. Ziolkowski, M. and Gratkowski, S. R., "Shielding from External Magnetic Fields by Rotating Nonmag- netic Conducting Cylindrical Shells," IEEE Transactions on Electromagnetic Compatability, Vol. 51, No. 3, August 2009, pp. 720-724.

21. Nijmeijer, H. and van der Schaft, A. J., Nonlinear Dynamical Control Systems, Springer-Verlag, New York, 1990, pp. 95-96.

22. US National Geospatial-Intelligence Agency, Technical Document for the World Magnetic Model (WMM), Released February 3, 2010. 